\title{
THE ASTEROID BELT AS A RELIC FROM A CHAOTIC EARLY SOLAR SYSTEM
}

\author{
André Izidoro $^{1,2}$, Sean N. Raymond ${ }^{1}$, Arnaud Pierens $^{1}$, Alessandro Morbidelli $^{3}$, \\ OTHON C. WINTER ${ }^{4}$, AND DAVID NeSVORNY̌ ${ }^{5}$ \\ ${ }^{1}$ Laboratoire d'astrophysique de Bordeaux, Université de Bordeaux, CNRS, B18N, allée Geoffroy Saint-Hilaire, F-33615 Pessac, France; izidoro.costa@gmail.com \\ ${ }^{2}$ Capes Foundation, Ministry of Education of Brazil, Brasília/DF 70040-020, Brazil \\ ${ }^{3}$ University of Nice-Sophia Antipolis, CNRS, Observatoire de la Côte d'Azur, Laboratoire Lagrange, BP 4229, F-06304 Nice Cedex 4, France \\ ${ }^{4}$ UNESP, Univ. Estadual Paulista-Grupo de Dinâmica Orbital \& Planetologia, Guaratinguetá, CEP 12.516-410, São Paulo, Brazil \\ ${ }^{5}$ Department of Space Studies, Southwest Research Institute, 1050 Walnut St., Suite 300, Boulder, CO 80302, USA \\ Received 2016 July 12; revised 2016 September 16; accepted 2016 September 19; published 2016 December 6
}

\begin{abstract}
The orbital structure of the asteroid belt holds a record of the solar system's dynamical history. The current belt only contains $\sim 10^{-3}$ Earth masses yet the asteroids' orbits are dynamically excited, with a large spread in eccentricity and inclination. In the context of models of terrestrial planet formation, the belt may have been excited by Jupiter's orbital migration. The terrestrial planets can also be reproduced without invoking a migrating Jupiter; however, as it requires a severe mass deficit beyond Earth's orbit, this model systematically under-excites the asteroid belt. Here we show that the orbits of the asteroids may have been excited to their current state if Jupiter's and Saturn's early orbits were chaotic. Stochastic variations in the gas giants' orbits cause resonances to continually jump across the main belt and excite the asteroids' orbits on a timescale of tens of millions of years. While hydrodynamical simulations show that the gas giants were likely in mean motion resonance at the end of the gaseous disk phase, small perturbations could have driven them into a chaotic but stable state. The gas giants' current orbits were achieved later, during an instability in the outer solar system. Although it is well known that the present-day solar system exhibits chaotic behavior, our results suggest that the early solar system may also have been chaotic.
\end{abstract}

Key words: chaos - minor planets, asteroids: general - planets and satellites: gaseous planets - planets and satellites: terrestrial planets

\section{INTRODUCTION}

The distribution of asteroids strongly constrains planet formation models. While the terrestrial planets' orbits are nearly circular and coplanar, the orbital eccentricities of asteroids are excited, filling parameter space from $e=0$ to 0.3 , and inclination $i=0^{\circ}$ to $20^{\circ}$. The asteroid belt's total mass is also only $\sim 10^{-3}$ Earth masses.

There are two basic views on how the inner solar system was built, with different implications for the asteroid belt. In one view, the asteroid belt contained a few Earth masses in solid material but was rapidly depleted and excited by dynamical mechanisms. Gravitational scattering of asteroids by a population of moon- to Mars-sized planetary embryos originally in the belt can promote significant depletion and excitation of the belt (Wetherill 1978, 1992, 1986; Chambers \& Wetherill 1998; Agnor et al. 1999; Petit et al. 1999; Chambers 2001; Petit et al. 2001, 2002, pp. 711-723; O'Brien et al. 2007). One problem with this scenario in the context of terrestrial planet formation is that Mars analogs produced in these simulations are far more massive than the actual one (e.g., Raymond et al. 2004; O'Brien et al. 2006; Raymond et al. 2006; Morishima et al. 2008; Raymond et al. 2009; Izidoro et al. 2013; Lykawka \& Ito 2013; Fischer \& Ciesla 2014; Izidoro et al. 2014). The Grand Tack scenario (Walsh et al. 2011) — which invokes the inward then outward migration of Jupiter through the asteroid belt region-removes enough mass beyond 1 au to explain why Mars is much smaller than Earth and to sculpt the asteroid belt in a way that will become consistent with its current structure via subsequent dynamical evolution (Roig \& Nesvorný 2015; Deienno et al. 2016). In the opposite view, the asteroid belt had low mass even at early times (Izidoro et al. 2015b; Levison et al. 2015; Moriarty \& Fischer 2015; Drazkowska et al. 2016), and Jupiter and Saturn did not migrate across the asteroid belt. In this framework, a primordial low-mass asteroid belt should be far less dynamically excited than the observed one (Izidoro et al. 2015b), and what remains to be explained is the belt's dynamical excitation (for a recent review, see Morbidelli et al. 2015, pp. 493-507).

In this paper we propose a novel mechanism for explaining the dynamical excitation of the asteroid belt. The mechanism relies on the chaotic evolution of Jupiter's and Saturn's orbits at early times. In Section 2 we present an example for the origin of chaos in Jupiter's and Saturn's early orbits. In Section 3 we present our results for the chaotic excitation of the asteroid belt. In Section 4 we more fully address the possible origins of chaos in the giant planets' orbits, and present alternative scenarios to trigger chaos. In Section 5 we discuss the implication of our results for models of solar system formation. Finally, in Section 6 we briefly summarize our findings.

\section{AN EXAMPLE OF CHAOS IN JUPITER'S AND SATURN'S EARLY ORBITS}

The excitation of the asteroid belt took place after the gaseous protoplanetary disk had dissipated, yet it is during the disk phase that the gas giants' orbits could have changed most dramatically due to orbital migration. Embedded in the gaseous disk, Jupiter and Saturn systematically migrate into mean motion resonance (MMR), where their orbital periods are related by a ratio of small integers (Masset \& Snellgrove 2001; Morbidelli \& Crida 2007; Pierens \& Nelson 2008; D’Angelo \& Marzari 2012; Pierens et al. 2014). The most common are the 3:2 and 2:1 MMRs. The present-day orbits of the giant planets 


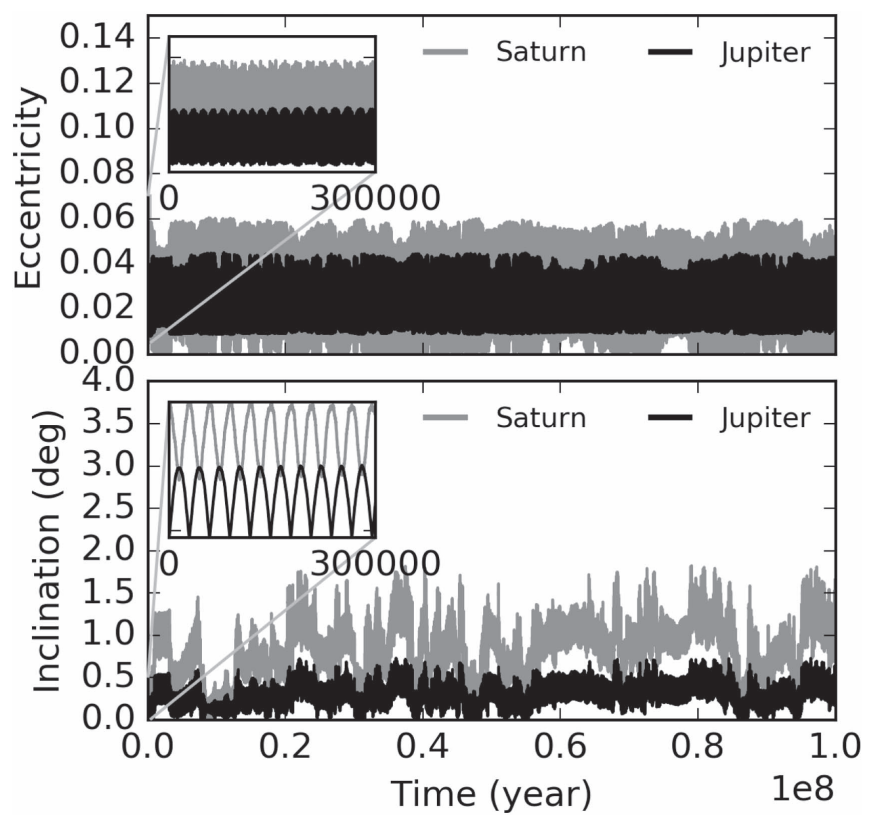

Figure 1. Onset of chaos in a characteristic dynamical simulation. Jupiter and Saturn started in the JSREG configuration, locked in 2:1 MMR with low eccentricities (starting eccentricities of 0.025 for both planets) and exhibiting regular motion. The evolution of their orbital eccentricities/inclinations are shown in the top/bottom panel. Gravitational scattering of a Mars-mass embryo initially at 12 au triggered chaos in the giant planets' orbits. The planets remained in resonance, as confirmed by libration of the $2: 1 \mathrm{MMR}$ critical angle $2 \lambda_{\text {Sat }}-\lambda_{\text {Jup }}-\varpi_{\text {Jup }}$, on chaotic but long-term stable orbits. $\lambda_{\text {Sat }}$, $\lambda_{\text {Jup }}$, and $\varpi_{\text {Jup }}$ are Saturn's mean longitude, Jupiter's mean longitude and longitude of pericenter, respectively. The initial conditions of this simulation are provided in the Appendix.

were achieved later, after the gaseous disk was gone, during a dynamical instability (Nesvorný \& Morbidelli 2012). In our model, the asteroids were excited between the dissipation of the disk and the instability.

In hydrodynamical simulations, Jupiter's and Saturn's migration typically leads to deep capture in resonance, with orbits characterized by regular motion. However, very small perturbations may push them into chaos (Sándor \& Kley 2006; Batygin \& Morbidelli 2013). Perturbations come from (i) dispersal of the gaseous disk and the corresponding loss of its damping (Papaloizou \& Larwood 2000; Cresswell \& Nelson 2008); (ii) gravitational forcing from the ice giants, both during their inward migration (Izidoro et al. 2015a) and after the dissipation of the gaseous disk; and (iii) gravitational scattering of small remnant planetary embryos and planetesimals in the giant planet region.

We performed a suite of numerical experiments to show that seemingly trivial perturbations can trigger chaos in the gas giants' orbits. Figure 1 shows one simulation in which a regularly evolving configuration of Jupiter and Saturn (the JSREG simulation), locked in 2:1 resonance, became chaotic as a result of the ejection of a Mars-sized embryo from the system. To perform this simulation we used the Mercury integrator (Chambers 1999). The system was composed by the central solar mass star, the fully formed Jupiter and Saturn in the 2:1 MMR, and a Mars-mass planetary embryo. Jupiter was initially at $5.25 \mathrm{au}$, Saturn at $\sim 8.33 \mathrm{au}$, and the planetary embryo at $12 \mathrm{au}$. The gas giants' eccentricities were initially 0.025 and their mutual orbital inclination $0^{\circ} .5$. The planetary embryo started with zero orbital eccentricity and inclination. We used an integration time step of 100 days and assumed that the gaseous disk was already fully dissipated.

While the planetary embryo in Figure 1 was only $1 / 4000$ the combined mass of the gas giants, it triggered chaos. The gas giants' orbits were chaotic but dynamically stable for long timescales, consistent with a late dynamical instability that rearranged their orbits (Tsiganis et al. 2005; Morbidelli et al. 2007; Levison et al. 2011). The perturbed giant planets even remained in 2:1 resonance with modest (but chaotic) eccentricities and inclinations (see for example Figure 2). Depending on the nature and strength of the perturbation, chaos was generated in up to $100 \%$ of our simulations starting from a regular, resonant configuration. In fact, stochastic forcing from turbulent density fluctuations during the disk phase may have pushed the planets out of (deep) resonance (Adams et al. 2008; Lecoanet et al. 2009; Pierens et al. 2011), onto nearby orbits where the density of chaos is high (see the Appendix).

This simulation (Figure 1) represents a simple proof of concept; a regular orbital configuration of Jupiter and Saturn can easily be converted into a chaotic one. The perturbation required must be strong enough to transition the system to a new dynamical state but not so strong as to make the system dynamically unstable. The magnitude of the perturbation in the simulation from Figure 1 is entirely plausible, as it is likely that some leftovers remained when the protoplanetary disk dissipated. Indeed, the so-called "late veneer" represents geochemical evidence that planetary leftovers remained scattered throughout the inner solar system after the Moonforming impact on Earth, long after the dissipation of the disk (e.g., Day et al. 2007; Walker 2009; Bottke et al. 2010; Jacobson et al. 2014). In Section 4 we present additional scenarios for generating chaos in Jupiter's and Saturn's early orbits.

\section{CHAOTIC EXCITATION OF THE ASTEROID BELT}

We now turn our attention to showing how the asteroids' orbits may have been excited by chaos in the orbits of Jupiter and Saturn. We used the Symba (Duncan et al. 1998), Swift (Levison \& Duncan 1994), Mercury (Chambers 1999), and Rebound (Rein \& Spiegel 2014; Rein \& Tamayo 2015) integrators to perform our simulations of the belt excitation. Asteroids were modeled as massless test particles. The integration time step in all our simulations was at most $1 / 20$ of the orbital period of the innermost body in the system. We stress that we are aware of an issue with Symba5 which compromises the performance of the integrator in the case where planets have close encounters every orbital period. When this kind of evolution takes place it degrades the symplectic nature of the integrator, causing large errors. This may be the case for example when Jupiter and Saturn evolve in a compact resonant configuration (e.g., 3:2 MMR). To make sure the chaos observed in our simulations has no numeric origin we tested an ample number of integrators over the different simulations presented here. In Rebound we test both the WHFAST and IAS15 integrators and in Mercury we performed tests with the Bulirsch-Stoer and "Hybrid" integrators.

Figure 2 shows the dynamical evolution of Jupiter and Saturn in the JSREG and JSCHA simulations, which we use as fiducial cases to illustrate and contrast mechanisms of excitation of the asteroid belt. These two simulations were generated from almost identical initial orbital arrangements; the 

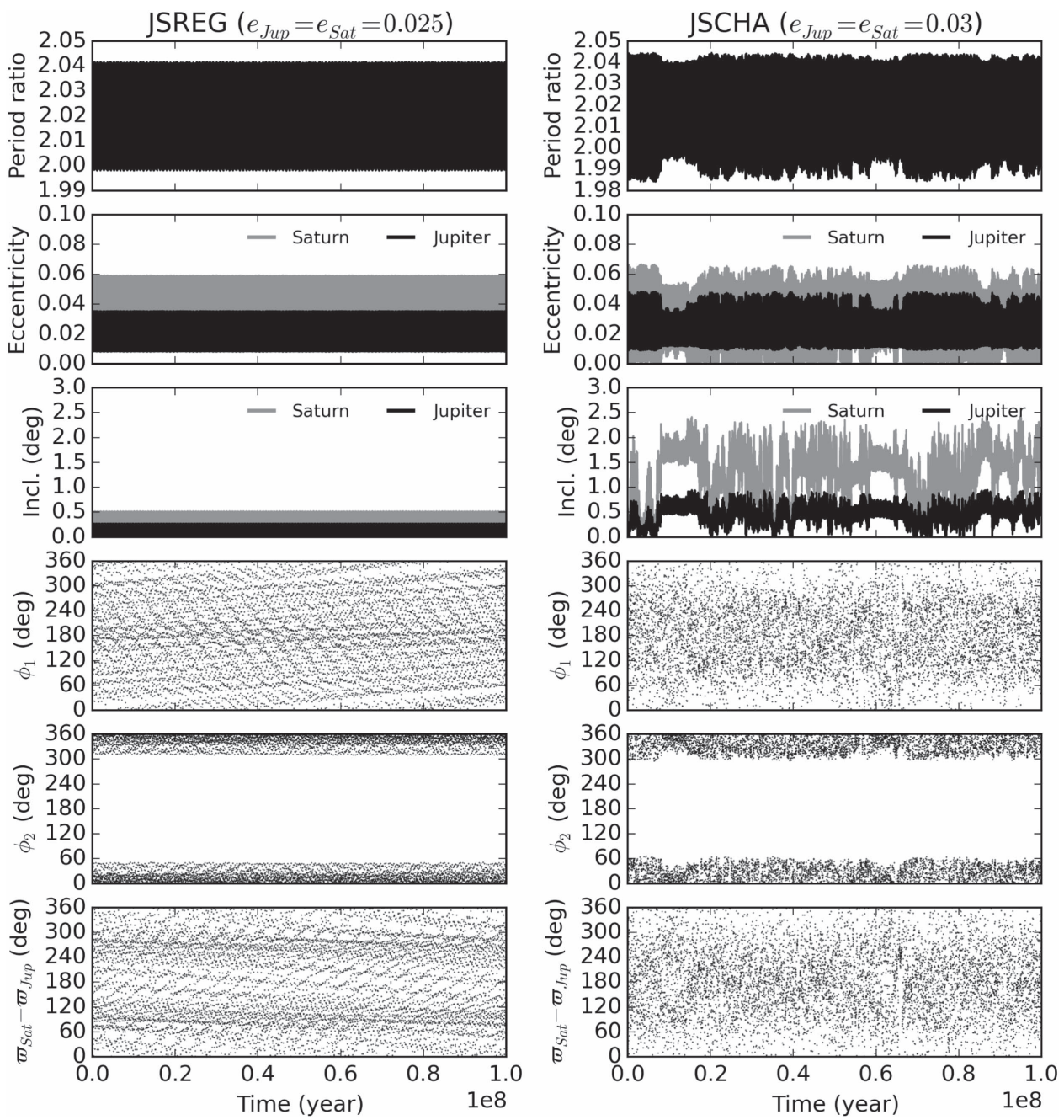

Figure 2. Dynamical evolution of regular Jupiter and Saturn in the JSREG and JSCHA simulations. In both cases the planets are in 2:1 MMR (the critical angle $\phi_{2}=2 \lambda_{\text {Sat }}-\lambda_{\text {Jup }}-\varpi_{\text {Jup }}$ associated with the 2:1 MMR librate around zero degree while the $\phi_{1}=2 \lambda_{\text {Sat }}-\lambda_{\text {Jup }}-\varpi_{\text {Sat }}$ circulates). $\lambda_{\text {Sat }}$ and $\varpi_{\text {Sat }}$ are Saturn's mean longitude and longitude of pericenter. $\lambda_{\text {Jup }}$ and $\varpi_{\text {Jup }}$ are Jupiter's mean longitude and longitude of pericenter. The initial conditions of these two simulations are provided in the Appendix.

only difference is that Jupiter and Saturn's eccentricities were each set to 0.025 in the JSREG simulation (resulting in regular motion) and to 0.03 in the JSCHA simulation (which triggered chaos). In both simulations, Jupiter and Saturn's semimajor axes are initially 5.4 and about $\sim 8.57$ au, respectively. Their mutual initial orbital inclinations are 0.5 . Their argument of pericenter and longitude of the ascending node are set zero. Jupiter's mean anomaly is initially zero and Saturn's mean anomaly is initially $180^{\circ}$.

Figure 2 shows the evolution of the gas giant's period ratio, eccentricities, orbital inclinations, and an angle associated with the 2:1 MMR between the planets. In both simulations, the planets are in 2:1 resonance since the critical angle $\phi_{2}=2 \lambda_{\text {Sat }}-\lambda_{\text {Jup }}-\varpi_{\text {Jup }}$ librates around zero degree, where $\lambda_{\text {Jup }}$ and $\lambda_{\text {Sat }}$ are the mean longitudes of Jupiter and Saturn, respectively. The critical angle $\phi_{1}=2 \lambda_{\text {Sat }}-\lambda_{\text {Jup }}-\varpi_{\text {Sat }}$ circulates, where $\varpi_{\text {Sat }}$ is Saturn's longitude of pericenter. Thus, the planets are not in apsidal corotation resonance-in which the planets undergo apsidal libration as well as libration of both resonant arguments (Michtchenko et al. 2008) - as shown by the circulation of the angles $\varpi_{\text {Sat }}-\varpi_{\text {Jup }}$ (Figure 2, bottom panels). Yet the eccentricity and inclination evolution of Jupiter and Saturn are quite different in the the two simulations. In particular, there are larger variations of eccentricity and inclination over $100 \mathrm{Myr}$ in the chaotic case. These variations are linked with the precession of longitudes, which translate to shifting resonances and gravitational perturbations in the belt.

We now show how, once the gaseous disk was gone, chaos in Jupiter's and Saturn's early orbits may have excited the asteroids' orbits even if the belt's primordial mass was very low (comparable to its current mass). The asteroid belt is speckled with resonances, locations where there is an integer match between the characteristic orbital frequencies of asteroids and the giant planets. MMRs are located where an asteroid's orbital period forms an integer ratio with a planet's period (Jupiter in this case). In secular resonances (SRs), a quantity related to the precession of an asteroid's orbit matches one of the giant planets. The most important SRs in the main belt are the $\nu_{6}$ and $\nu_{16}$ resonances, where an asteroid's apsidal and nodal precession rate (or frequency), respectively, match that of Saturn (Froeschle \& Scholl 1989; Morbidelli \& Henrard 1991). When Jupiter's and Saturn's orbits evolve in a regular fashion, MMRs and SRs are stationary, so asteroids in certain parts of the belt are excited whereas asteroids in other parts are not (Morbidelli \& Henrard 1991). 

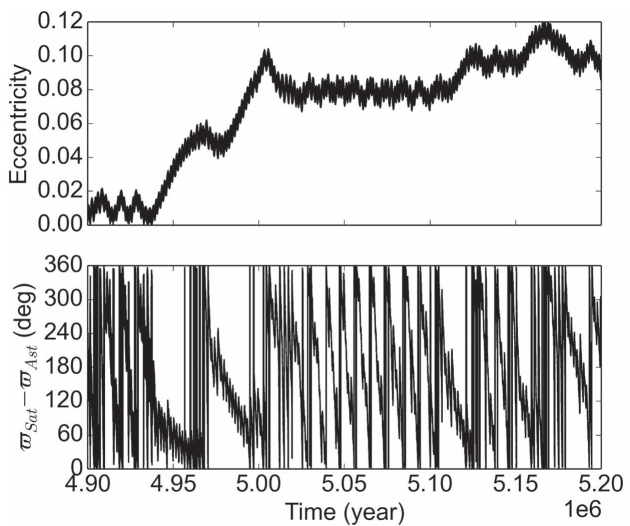
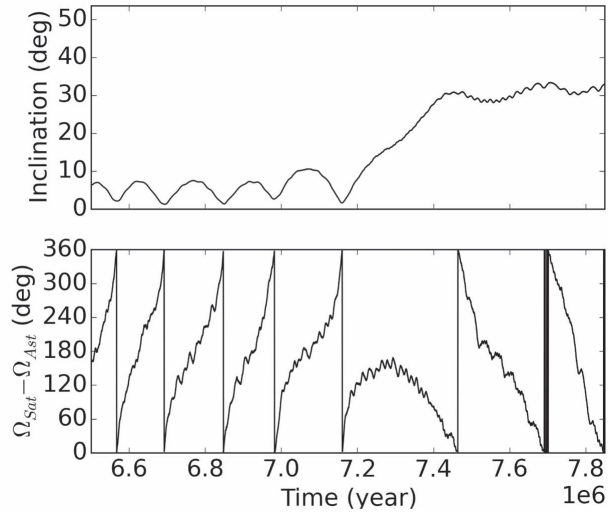

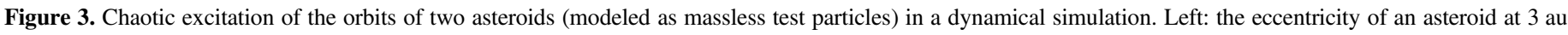

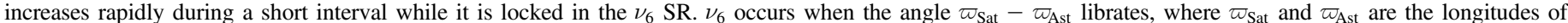

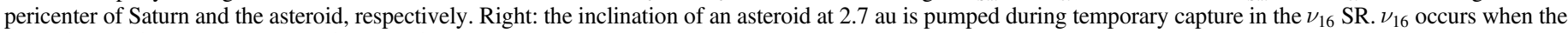

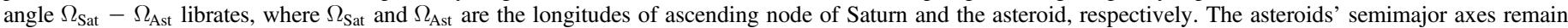
roughly constant.

When the giant planets evolve chaotically, their orbital alignments undergo stochastic jumps, i.e., they may precess at many different frequencies and their orbits may even have its direction of apsidal precession temporarily reversed. The location of SRs within the belt undergo corresponding jumps (MMRs are much less sensitive to such variations). When a resonance jumps to the location of a given asteroid its orbit is significantly excited on a relatively short $\left(\sim 10^{4-6}\right.$ year $)$ timescale. Figure 3 shows how the $\nu_{6}$ and $\nu_{16}$ SRs pump the eccentricity and orbital inclinations, respectively, of two asteroids. Note that the time intervals shown in both plots of Figure 3 were purposely chosen to clearly illustrate the effect of the respective resonances. The lifetime of a particle in the belt depends on the chaotic evolution of the gas giants and the particle's initial orbit. We discuss and show in the Appendix that the effects of the chaotic excitation can also eject particles from the system and even empty parts of the belt.

In addition to $\nu_{6}$ and $\nu_{16}$, other resonances also play a role in pumping asteroids' eccentricities and/or inclinations (see the Appendix). These include resonances resulting from the linear combination of principal secular frequencies (nonlinear secular resonances), MMRs, secondary resonances (linear combination between the main secular and short period frequencies associated with orbital period), and Kozai resonances. Another factor is that, unlike MMRs, which are linked to a given orbital radius, the locations of SRs are a function of an asteroid's proper inclination and eccentricity (Froeschle \& Scholl 1989; Morbidelli \& Henrard 1991).

We can understand the chaotic excitation of asteroids using a Fourier analysis of Saturn's longitude of pericenter $\varpi_{\text {Sat }}$ (Figure 4; top panels). When Jupiter and Saturn undergo regular motion, the power spectrum of $\varpi_{\text {Sat }}$ is peaked, dominated by characteristic frequencies (in particular the $\mathrm{g}_{6}$ frequency at $\sim 1 / 62000 \mathrm{yr}^{-1}$, which controls the location of the $\nu_{6}$ SR) and their harmonics (Laskar 1990, 1993; Michtchenko \& Ferraz-Mello 1995). The peaked nature of the power spectrum indicates that Saturn's precession frequency-and thus the location of the $\nu_{6}$ and other resonances-is fixed. This explains why eccentricities and inclinations of asteroids are only strongly excited at specific locations in the belt (Figure 4). In contrast, the power spectrum of a simulation in which
Jupiter's and Saturn's orbits evolve chaotically (the JSCHA simulation) shows a broad band of frequencies instead of a few strong peaks. In this case the SR $\nu_{6}$ jumps across the entire belt because $\varpi_{\text {Sat }}$ precesses at many different frequencies due to Saturn's chaotic interactions with Jupiter. Note from the frequency analysis in Figure 4 that in the JSREG simulation the longitude of pericenter of Saturn precesses (slowly) positively while the longitude of pericenter of Jupiter precesses (quickly) backwards. If planets were in apsidal corotation, that would imply both their longitudes of pericenter would precess in the same direction (negative). Because asteroids suffering secular perturbation always precess positively, $\nu_{6}$ could not exist in the belt if the asteroids' and Saturn's longitude of pericenter precess in different directions.

During the gas giants' chaotic evolution the $\nu_{6}$ SR jumps across the entire asteroid belt. To estimate the radial extent of the jumps of $\nu_{6}$ we first compute the precession frequency that an asteroid would have with Jupiter and Saturn in 2:1 resonance. Using linear secular theory (see Murray \& Dermott 1999), this frequency is given by

$$
\begin{aligned}
A= & \frac{1}{8 \pi} n_{\text {Ast }}\left[\frac{M_{\text {Jup }}}{M_{\odot}}\left(\frac{a_{\text {Ast }}}{a_{\text {Jup }}}\right)^{2} b_{3 / 2}^{(1)}\left(\alpha_{\text {Jup }}\right)\right. \\
& \left.+\frac{M_{\text {Sat }}}{M_{\odot}}\left(\frac{a_{\text {Ast }}}{a_{\text {Sat }}}\right)^{2} b_{3 / 2}^{(1)}\left(\alpha_{\text {Sat }}\right)\right],
\end{aligned}
$$

where $\mathrm{n}_{\mathrm{Ast}}$ and $a_{\mathrm{Ast}}$ are the mean motion and semimajor axis of the asteroid. $M_{\odot}, M_{\mathrm{Jup}}$, and $M_{\mathrm{Sat}}$ are the solar mass, Jupiter's mass and Saturn's mass, respectively. $a_{\text {Jup }}$ and $a_{\text {Sat }}$ are Jupiter's and Saturn's semimajor axes, respectively. $b_{3 / 2}^{(1)}$ is the Laplace coefficient which is computed as a function of $\alpha_{\text {Jup }}$ and $\alpha_{\text {Sat }}$, which are given by

$$
\begin{aligned}
& \alpha_{\mathrm{Jup}}=\frac{a_{\mathrm{Ast}}}{a_{\mathrm{Jup}}} \\
& \alpha_{\mathrm{Sat}}=\frac{a_{\mathrm{Ast}}}{a_{\mathrm{Sat}}}
\end{aligned}
$$

If we assume that $\varpi_{\text {Sat }}$ precesses with frequencies between $\sim 10^{-5}$ and $\sim 10^{-4}$ /year the location of $\nu_{6}$ varies from about 1.4 to 3.5 au (Figure 5). The asteroids' eccentricities are thus 

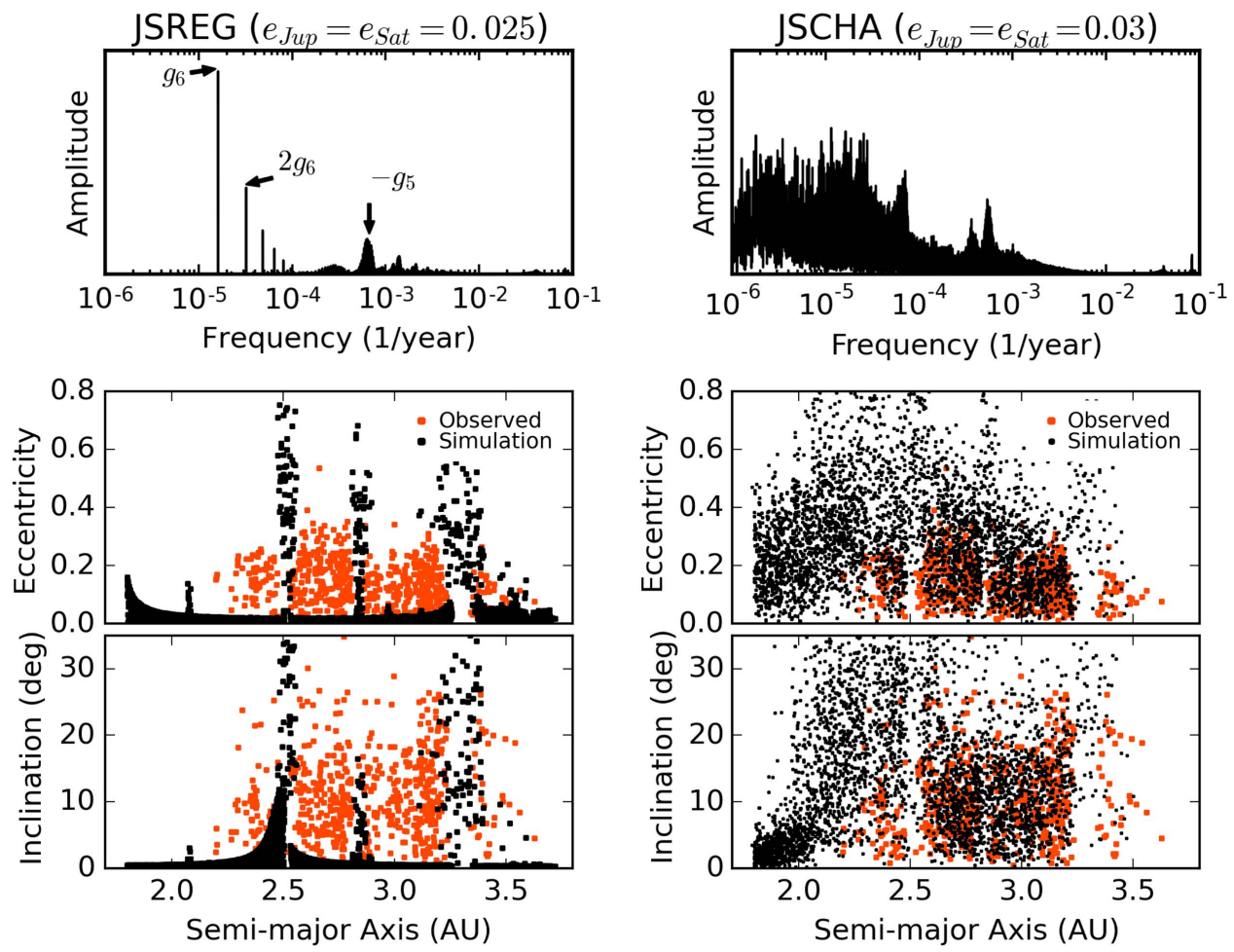

Figure 4. Fourier analysis and excitation of the asteroid belt by the gas giants in two $N$-body simulations. In both cases, Jupiter and Saturn are locked in 2:1 MMR, with starting semimajor axes of 5.25 au and $\sim 8.33$ au. In the JSREG simulation (left panels) the gas giants' initial eccentricities are 0.025 and their orbits exhibit regular motion. In the JSCHA simulation (right) the giants' initial eccentricities are 0.03 and their orbits are chaotic. Their mutual orbital inclination is initially 0.5 . Each system was integrated for $136 \mathrm{Myr}$, and to perform the Fourier analysis we used an output time step of 2 years; the top panels show the power spectra for $\varpi_{\text {Sat }}$ for each case. The middle and bottom panels show the dynamical excitation of the main belt in the two simulations, using a snapshot at 40 Myr. Some asteroids in the JSCHA simulation have larger eccentricities and inclinations than those observed. This does not mean that our results are inconsistent with the present-day asteroid belt. Highly eccentric and inclined objects are removed from the system during the later evolution of the solar system: during the dynamical instability between the giant planets (Morbidelli et al. 2010) and over the subsequent 3.8 billion years (Minton \& Malhotra 2010). These simulations also do not take into account the gravitational influence of the growing terrestrial planets, which may remove a large fraction of dynamically overexcited asteroids. In the JSCHA simulation, asteroids with low orbital inclination are also observed between 1.8 and $2 \mathrm{au}$. Equally, these objects do not exist in the real belt today. It is highly likely that these objects will also be removed from this region during the accretion of the terrestrial planets and by the effects of SRs when Jupiter and Saturn reach their current orbits (e.g., $\nu_{16}$ is at $\sim 1.9$ au today; Froeschle \& Scholl 1989).

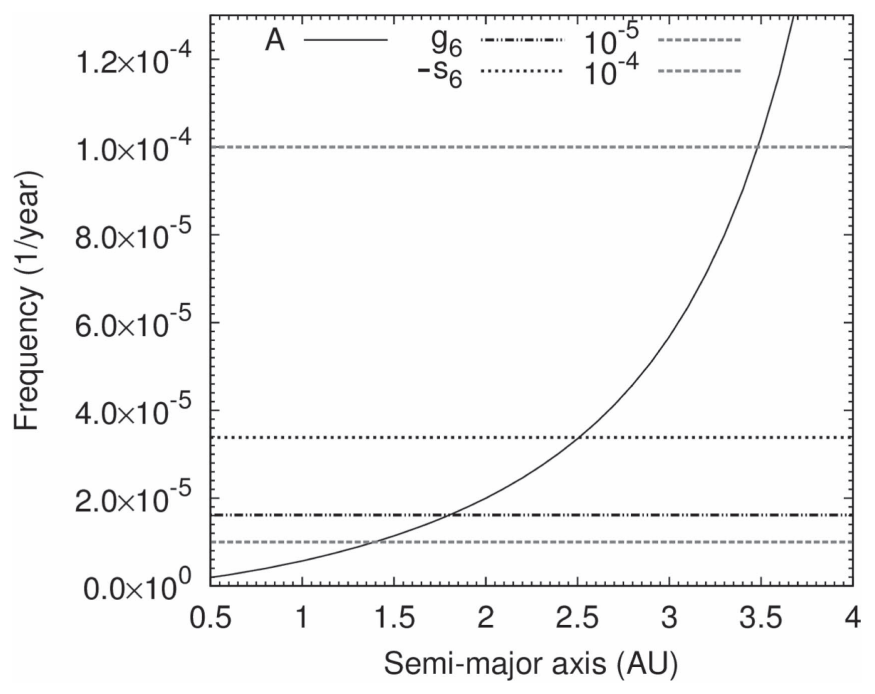

Figure 5. Precession frequency of asteroids at different distances from the star calculated from linear secular theory in the dynamical system including Jupiter and Saturn. The gas giants are initially as in the JSREG simulation. The $\nu_{6}$ and $\nu_{16}$ locations in the JSREG simulation corresponds to the intersection between the A curve and the $g_{6}$ and $s_{6}$ frequencies, respectively. Frequencies corresponding to $10^{-5}$ to $10^{-4} \mathrm{yr}^{-1}$ are shown to estimate how much $\nu_{6}$ jumps across the belt in the JSCHA simulation. excited across the entire main belt. An analogous process acts to pump the asteroids' inclinations. In the JSCHA simulation, although $\nu_{16}$ is much stronger and wider than in the JSREG simulation, it does not jump across the entire belt. However, other resonances contribute to exciting the asteroids' inclinations across the entire belt (see the Appendix).

The timescale for chaotic excitation of the full asteroid belt is a few million years to hundreds of millions of years depending on the evolution of the gas giants (additional examples are given in the Appendix). The surviving asteroids broadly match the observed distribution (Figure 4; right-hand side, middle and bottom panels). In simulations that successfully excited the asteroid belt, two conditions were typically observed. First, Jupiter's and Saturn's orbits were chaotic in both their eccentricities and inclinations (Barnes et al. 2015). The hardest aspect of the asteroid belt to reproduce is its broad inclination distribution (Izidoro et al. 2015b). Second, Jupiter's eccentricity was not too high. In simulations in which Jupiter's eccentricity remained much larger than its current value of $\sim 0.05$ for longer than $100 \mathrm{Myr}$, parts of the belt were emptied. Finally, although we have illustrated this mechanism with Jupiter and Saturn in 2:1 MMR, we observe chaotic excitation in a number of resonant (or near-resonant) orbits, including the 2:1, 3:2, 7:4, and 5:3 (see the Appendix). 


\section{PATHS TO CHAOS IN JUPITER'S AND SATURN'S EARLY ORBITS}

We have argued that Jupiter's and Saturn's orbits may have evolved chaotically at early times. In this section we further justify this argument. We first map the prevalence of chaotic motion in the phase space in the orbits of Jupiter and Saturn. Next we perform a series of numerical experiments to mimic the orbital migration and resonant capture of Jupiter and Saturn while they were embedded in the gaseous disk. After the disk's dissipation, a large number of simulations exhibited chaotic behavior. Finally, we show the long-term dynamical stability between the giant planets evolving in a chaotic resonant configuration.

\subsection{A Map of Chaos in Jupiter's and Saturn's Orbits}

To get a sense of the presence of chaos across the phase space of Jupiter's and Saturn's orbital configuration, we performed about 9000 simulations to build a dynamical map for a wide range of orbital period ratios between Jupiter and Saturn. The MEGNO (Mean Exponential Growth factor of Nearby Orbits; Cincotta \& Simo 2000) chaos indicator is a powerful tool used to identify chaos in dynamical systems. Chaotic orbits are characterized by a large MEGNO value $(\langle Y\rangle \gg 2)$ while regular or quasi-periodic orbits are associated with $\langle Y\rangle \rightarrow 2$ (e.g., Cincotta \& Simo 2000).

Our initial conditions were set as follows. Jupiter was placed at 5.25 au while Saturn's semimajor axis was sampled from about 6.25 to 8.6 au (period ratio between 1.3 and 2.1). Their mutual inclination was sampled randomly from $0^{\circ}$ to 0.5 . The eccentricity of Jupiter was randomly selected between 0 and 0.01 . Saturn's initial eccentricity ranges from 0 to 0.1 . Note that although the initial eccentricity of Jupiter in this set of simulations is smaller than the corresponding initial values in Figures 1 and 2, this quantity does not remain constant over time. Jupiter and Saturn's (secular) interaction leads to eccentricity oscillations that imply that Jupiter's eccentricity may reach values comparable to or even larger than 0.025-0.03. Angular orbital elements of both planets were all sampled randomly between $0^{\circ}$ and $360^{\circ}$. Simulations were integrated for 50 Myr using the REBOUND integrator (Rein \& Tamayo 2015) computing the MEGNO value of each of these dynamical states.

Figure 6 shows a dynamical map of the behavior of Jupiter's and Saturn's orbits at different orbital separations. The simulations were integrated for fifty million years and the results are color-coded by MEGNO value. Black regions are potentially unstable; they show orbital configurations where Jupiter and Saturn undergo close encounters. Orange regions exhibit chaotic motion (with $\langle Y\rangle \gtrsim 4$ ) while blue regions encloses regular motion (with $\langle Y\rangle=2$ ). Chaotic regions are generally confined to orbital period ratios and are associated with specific MMRs. There is a broad chaotic region near the 2:1 MMR and narrower regions close to the 5:3 (period ratio of 1.66) and 7:4 (period ratio of 1.75) resonances, with some chaos present just exterior to the $3: 2$ resonance (period ratio of 1.5$)$.

The dynamical map in Figure 6 shows that chaos is common in the phase space available for Jupiter's and Saturn's orbits. Yet their actual orbits at early times were not chosen at random. Rather, the gas giants' orbital configuration was generated by interactions between the growing planets and the gaseous

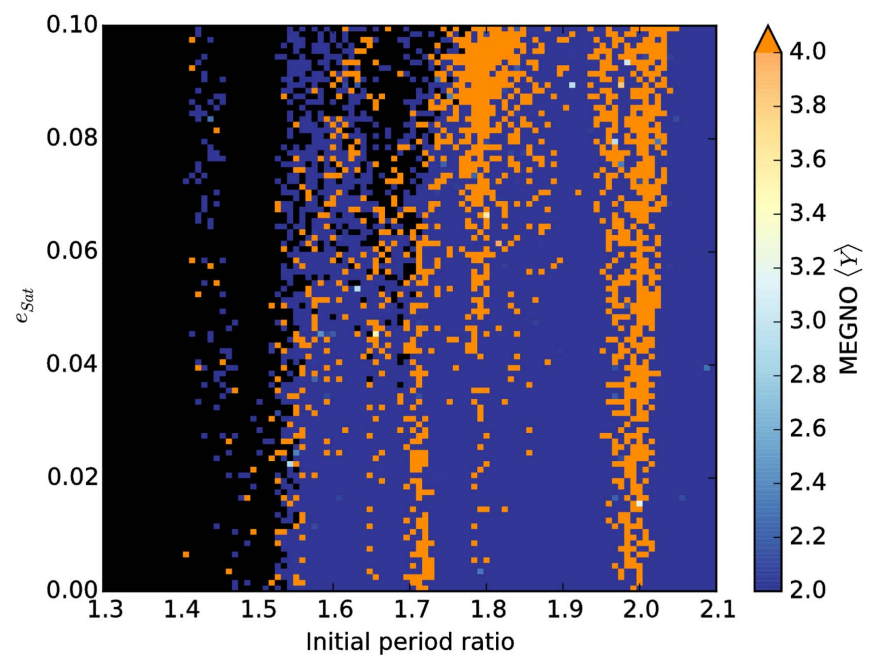

Figure 6. A dynamical map of chaos in Jupiter's and Saturn's orbits as a function of their initial orbital period ratio. The vertical axes show the initial eccentricity of Saturn (Jupiter's initial eccentricity was randomly chosen between zero and 0.01). The horizontal axes show the initial period ratio between Jupiter and Saturn. Each dynamical state is color-coded showing the MEGNO value after $50 \mathrm{Myr}$ of integration. Black is used to denote orbits where Jupiter and Saturn's mutual distance gets smaller than 1 au. Because we use a symplectic integrator, these orbits would not be solved properly in this case. Bluish colors show regular or quasi-periodic motion while orange-ish colors show chaotic orbits.

protoplanetary disk, in particular by a combination of orbital migration (Lin \& Papaloizou 1986; Ward 1997) and eccentricity and inclination damping (e.g., Papaloizou \& Larwood 2000; Bitsch et al. 2013). The dynamical evolution of Jupiter and Saturn in the gas disk depends on the disk properties (Masset \& Snellgrove 2001; Morbidelli \& Crida 2007; Zhang \& Zhou 2010; Pierens \& Raymond 2011). To study chaos in Jupiter's and Saturn's orbits in the context of orbital migration we perform $N$-body simulations using artificial forces to mimic the effects of the gas and also pure hydrodynamical simulations. We present these results next.

\subsection{Chaos in Jupiter's and Saturn's Orbits in the Context of Orbital Migration}

In our simulations of Jupiter and Saturn migrating in a gaseous disk, Jupiter was initially at 5.25 au and Saturn was initially placed exterior to the 2:1 MMR with Jupiter (beyond $8.33 \mathrm{au}$ ). Following Baruteau et al. (2014, p. 4293), Jupiter was assumed to migrate in a type-II mode with the migration timescale computed as

$$
t_{m, \text { Jup }}=\frac{2 a_{\text {Jup }}^{2}}{3 \nu} \times \min \left(1, \frac{M_{\text {disk }}}{m_{\text {Jup }}}\right) \text {, }
$$

where $a_{\mathrm{Jup}}$ and $m_{\mathrm{Jup}}$ are Jupiter's semimajor axis and mass, respectively. $\nu$ is the gas viscosity and $M_{\text {disk }}$ is the gas disk mass. We modeled the disk viscosity using the standard "alpha" prescription given by $\nu=\alpha c_{s} H$ (Shakura \& Sunyaev 1973), where $c_{s}$ is the isothermal sound speed and $H$ is the disk scale height. In our simulations $\alpha=0.002$ and the disk aspect ratio is $h \sim 0.07$. To account for the damping of eccentricity and inclination on Jupiter's orbit we assume the following relationship between migration timescale and 
eccentricity/inclination damping (Crida et al. 2008):

$$
t_{e, \text { Jup }}=t_{i, \text { Jup }}=t_{m, \text { Jup }} / K \text {. }
$$

In our simulations, we generally adopted the typical value of $K=10$, although we also performed simulations with $K=1$ and $K=100$.

To mimic the migration of Saturn in the gas disk, for simplicity we use the type-I migration/damping approach (Papaloizou \& Larwood 2000; Tanaka et al. 2002; Tanaka \& Ward 2004; Cresswell \& Nelson 2006, 2008). Since Saturn's gap is not fully open, this is an acceptable approximation. We stress that our goal here is only to have convergent and smooth migration of Jupiter and Saturn such that we can access the plausibility of chaos origin in this kind of simulation. The initial gas surface density at the location of Saturn is $\Sigma_{\text {Sat }}=900 r_{\text {Sat }}^{-0.5} \mathrm{~g} \mathrm{~cm}^{-2}$. To implement migration, eccentricity, and inclination damping on Saturn, we use the following formulas:

$$
\begin{gathered}
t_{m, \text { Sat }}=\frac{2}{2.7+1.1 \beta}\left(\frac{M_{\odot}}{m}\right)\left(\frac{M_{\odot}}{\sum_{g} a^{2}}\right)\left(\frac{h}{r}\right)^{2}\left(\frac{1+\left(\frac{e r}{1.3 h}\right)^{5}}{1-\left(\frac{e r}{1.1 h}\right)^{4}}\right) \Omega_{k}^{-1}, \\
t_{e, \text { Sat }}=\frac{t_{\text {wave }}}{0.780}\left(1-0.14\left(\frac{e}{h / r}\right)^{2}+0.06\left(\frac{e}{h / r}\right)^{3}\right. \\
\left.+0.18\left(\frac{e}{h / r}\right)\left(\frac{i}{h / r}\right)^{2}\right)
\end{gathered}
$$

and

$$
\begin{aligned}
t_{i, \mathrm{Sat}}= & \frac{t_{\mathrm{wave}}}{0.544}\left(1-0.3\left(\frac{i}{h / r}\right)^{2}+0.24\left(\frac{i}{h / r}\right)^{3}\right. \\
& \left.+0.14\left(\frac{e}{h / r}\right)^{2}\left(\frac{i}{h / r}\right)\right)
\end{aligned}
$$

where

$$
t_{\text {wave }}=\left(\frac{M_{\odot}}{m}\right)\left(\frac{M_{\odot}}{\Sigma_{g} a^{2}}\right)\left(\frac{h}{r}\right)^{4} \Omega_{k}^{-1}
$$

and $M_{\odot}, a, m, i$, and $e$ are the solar mass, planet's semimajor axis, planet's mass, orbital inclination, and eccentricity, respectively. $r$ is the planet's heliocentric distance. $\Sigma_{g}$ and $\beta$ are the gas disk surface density and gas surface profile index at the planet's location, respectively. In our simulations, in the case of Saturn, $\beta$ was calibrated from hydrodynamical simulations. The synthetic accelerations to account for the effects of the gas on the planet were modeled as

$$
\begin{aligned}
& \boldsymbol{a}_{\mathrm{mig}}=-\frac{\boldsymbol{v}}{t_{m, \mathrm{pla}}} \\
& \boldsymbol{a}_{e}=-2 \frac{(\boldsymbol{v} \cdot \boldsymbol{r}) \boldsymbol{r}}{r^{2} t_{e, \mathrm{pla}}} \\
& \boldsymbol{a}_{i}=-\frac{v_{z}}{t_{i, \mathrm{pla}}} \boldsymbol{k},
\end{aligned}
$$

where $\boldsymbol{k}$ is the unit vector in the $z$-direction. The label "pla," which appears in Equations (8)-(10), takes the form "Jup" or

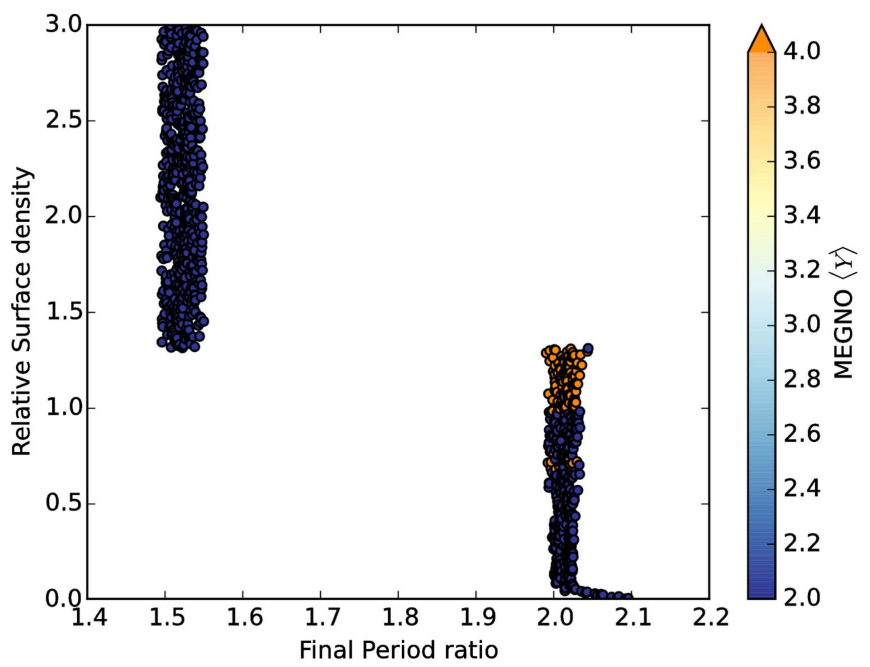

Figure 7. Final period ratio of Jupiter and Saturn migrating in $N$-body simulations including the effects of a 1D gas disk. The vertical axis shows the relative surface density of the disk. The gas disk lasts 1 Myr. After the gas dissipation, the orbits of the giant planets are numerically integrated for another $50 \mathrm{Myr}$. The color code shows the MEGNO value of each system at the end of the simulation. In this set of simulations, there is no case where Jupiter and Saturn suffer close encounters (there are no black points in the figure, which is different from Figure 6).

"Sat" to refer to accelerations applied to Jupiter and Saturn, respectively.

To ensure the robustness of this method, we compared selected simulations with true hydrodynamical simulations using similar disk setups and they agree in terms of the final period ratio, eccentricities, and orbital inclination of Jupiter and Saturn.

In simulations in which the ice giants were also included, they were assumed to migrate inward in the type-I mode described above.

We performed 1000 simulations that differed only in the initial surface density of the disk. In each case the gas disk was assumed to dissipate exponentially in $1 \mathrm{Myr}\left(\tau_{\mathrm{gas}}=100 \mathrm{kyr}\right)$. After the gas disk dissipated, simulations were integrated for another 50 Myr. To compute how chaotic the orbits of the giant planets are in each simulation we again used the MEGNO chaos indicator incorporated in the REBOUND integrator (Rein \& Tamayo 2015).

Figure 7 shows the results of these simulations. The vertical axis shows the relative initial surface density of the disk and the horizontal axis shows the final orbital period ratio between Jupiter and Saturn. Each final dynamical state of our simulation is represented with a circle whose color shows the MEGNO value of the final dynamical state of Jupiter and Saturn.

The experiment presented in Figure 7 produced more regular configurations of Jupiter and Saturn than chaotic ones. In agreement with pure hydrodynamical simulations (Zhang \& Zhou 2010; Pierens et al. 2014), Jupiter and Saturn typically park in either 3:2 (in high-mass disks) or 2:1 (low-mass disks) MMR. We did not find chaos in simulations where Jupiter and Saturn ends in 3:2. All our instances of chaos were related to Saturn and Jupiter's period ratio being close to 2 . We observe chaos in about $1 \%-25 \%$ of these simulations depending on the disk parameters and $K$-value (a parameter that defines the ratio between the migration timescale and eccentricity/inclination damping; see Equation (3)). We expect that chaotic configurations for Jupiter and Saturn in 3:2 may be more likely for larger 
eccentricities than our migration and damping prescriptions allow (see Figure 6).

We performed 15 hydrodynamical simulations using the GENESIS code (de Val-Borro 2006). None of the simulations with Jupiter and Saturn migrating in the disk generated chaos in Jupiter's and Saturn's orbits. However, both hydrodynamical simulations and our $N$-body simulations with synthetic forces are extremely idealized. A number of factors could change the outcome. First, the protoplanetary disks in these simulations are typically laminar and perfectly axisymmetric. Second, the gas disk's dissipation in numerical simulations is in general poorly modeled with an exponential density decay over the entire disk. Third, at the end of the gas disk phase, planets should evolve in a sea of planetesimals and leftover building blocks of their own process of formation. In these simulations we did not include any external perturbation in the system (but see Section 2). Fourth, the migrating ice giants (and/or their building blocks; Izidoro et al. 2015a) represent a further source of perturbations.

In fact, the results shown in Figures 6 and 7 are complementary in nature. The setup of the simulations in Figure 7 favor deep capture in resonance and regular motion between Jupiter and Saturn while that in Figure 6 (because of the random selection of angles) may preferentially put the dynamical state slightly off the libration center and favor mostly chaotic orbits. The existence of many chaotic configurations in Figure 6 demonstrates the prevalence of chaos and even if the gas giants' orbits behaved regularly immediately after the dissipation of the gaseous disk, there are a number of processes that could have rendered them chaotic.

\subsection{Long-term Dynamical Stability of the Gas Giants in a Chaotic Configuration}

The present-day orbits of the giant planets are thought to have been achieved by an instability in the giant planets' orbits that occurred long after the dissipation of the gases (Tsiganis et al. 2005; Morbidelli et al. 2007; Levison et al. 2011). Our model is entirely consistent with a late ( 500 Myr-later) instability in the giant planets' orbits, whatever the exact configuration of Jupiter and Saturn (see Batygin et al. 2012; Nesvorný \& Morbidelli 2012; Pierens et al. 2014). In fact, our model is also consistent with an earlier giant planet instability (Kaib \& Chambers 2016), as long as there is a sufficiently long interval during which the belt can be chaotically excited. This interval is roughly longer than $10 \mathrm{Myr}$ in most of the simulations we have run, but can be as short as $2 \mathrm{Myr}$. To illustrate the possibility of a long-term stability between the giant planets evolving in chaotic orbits, we performed $N$-body simulations in which Jupiter, Saturn, Uranus, and Neptune migrate in a disk. The prescription for migration used in these simulations is analogous to that explained before in this text. Isothermal type-I migration and damping are also applied to the ice giants. Here, we again used those damping timescales defined in Equations (4)-(7) and accelerations given by Equations (8)-(10). Figure 8 shows a long-term stable dynamical evolution of the gas giants in chaotic orbits. At the end of the gas disk phase, Jupiter and Saturn are locked in 2:1 MMR, Saturn-Uranus in 2:1 MMR, and Uranus-Neptune in 3:2 MMR. The system is dynamically stable for over $500 \mathrm{Myr}$.
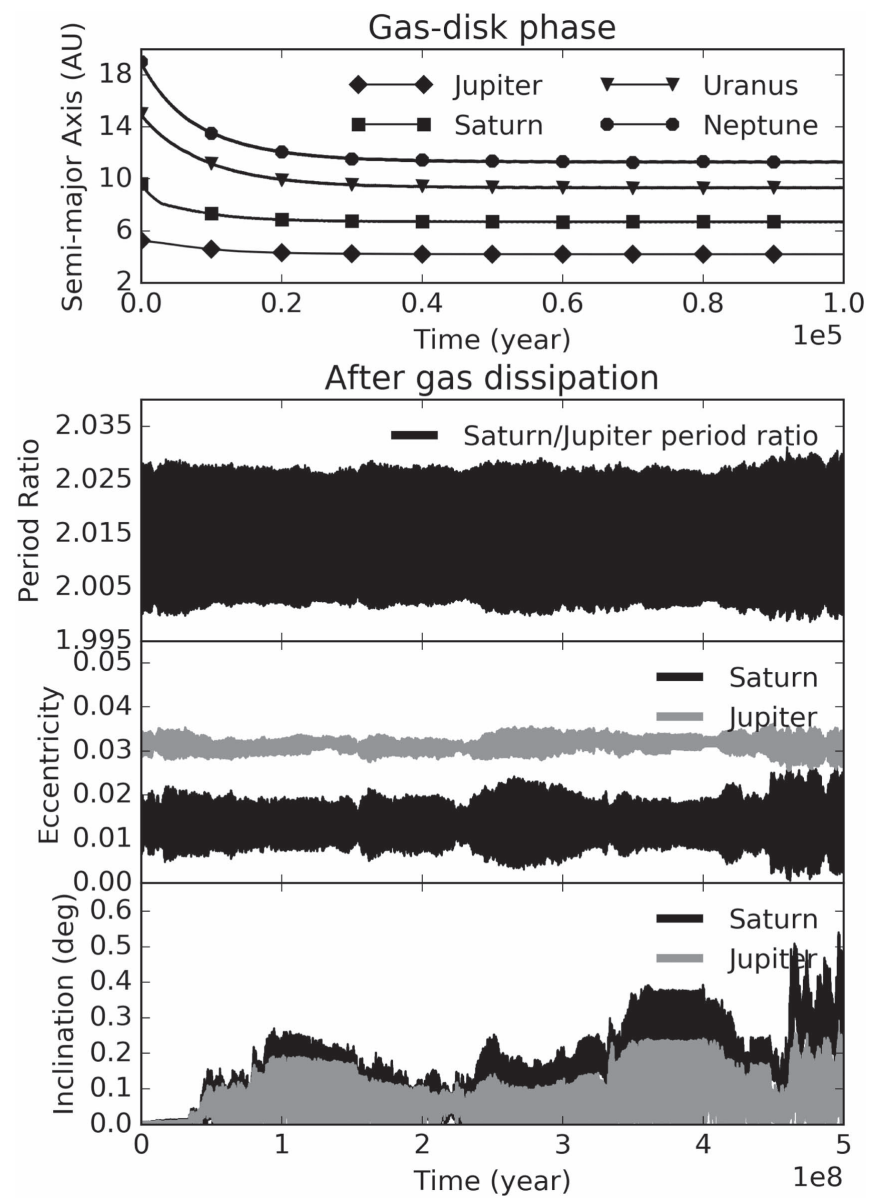

Figure 8. Example of the genesis of chaos in the giant planets' orbits and their long-term stability. Top: orbital migration of Jupiter, Saturn, Uranus, and Neptune embedded in the gaseous protoplanetary disk. At the end of the gas disk phase, the planets are locked in a chain of MMRs $(2: 1,2: 1,3: 2)$. Bottom: long-term evolution of the Saturn-to-Jupiter orbital period ratio, and the gas giants' eccentricities and inclinations over the next 500 Myr. This configuration is consistent with a later instability in the orbits of the giant planets (Tsiganis et al. 2005; Levison et al. 2011; Nesvorný \& Morbidelli 2012).

\section{DISCUSSION}

Chaotic excitation of the asteroid belt represents a novel mechanism for solving a longstanding problem in planetary science. Yet there are a number of questions that arise when considering whether this mechanism is consistent with our current vision of the solar system's global evolution.

In this section we address a number of questions.

\subsection{Chaotic Excitation versus the Grand Tack}

The "small Mars" problem highlights the fact that a mass deficit is needed from $\sim 1-4$ au to explain Mars' small mass relative to Earth's (Wetherill 1996; Raymond et al. 2009). In the Grand Tack model, this deficit is generated by Jupiter's long-distance orbital migration from several au to $1.5 \mathrm{au}$, then back out to beyond 5 au (Walsh et al. 2011; Jacobson \& Morbidelli 2014; Raymond \& Morbidelli 2014; Brasser et al. 2016)

By explaining the asteroid belt's orbital structure, our results revive models in which the asteroid belt was initially very low mass (Izidoro et al. 2015b; Levison et al. 2015). While standard disk models typically invoke a smooth mass distribution within disks (for example, the minimum-mass solar nebula model of 
Weidenschilling 1977 and Hayashi 1981 generates a smooth disk from discrete planets), it remains unclear whether the planetary building blocks embedded in these gaseous disks should really follow a smooth distribution. In fact, models often find that planetesimals preferentially grow in special locations within the disk, such as at pressure bumps (e.g., Johansen et al. 2014, p. 547). Some models naturally create confined rings of planetesimals within broad disks (Surville et al. 2016). There also exist mechanisms to systematically drain solids from certain areas of the disk, thereby creating localized depletions and enhancements (e.g., Levison et al. 2015; Moriarty \& Fischer 2015; Drazkowska et al. 2016).

Our model thus forms the basis of an alternative to the Grand Tack model. Within the context of this model, Mars' small mass can be explained by a broad mass depletion between Earth's and Jupiter's orbits. The asteroid belt, which could not be stirred by the dynamical effects of local embryos, was chaotically excited by Jupiter and Saturn.

The next step is to search for ways to distinguish between the Grand Tack and this new chaotic model. Tests may be based on observations of small bodies in the solar system or geochemical measurements. Alternately, the models may be differentiated by more detailed studies of the underlying physical mechanisms involving planetary orbital migration and pebble accretion.

Like the Grand Tack, our model assumes that Jupiter and Saturn migrated during the gas disk phase into a resonant configuration, mostly likely to $3: 2$ or $2: 1$ MMR (Masset \& Snellgrove 2001; Morbidelli \& Crida 2007; Pierens \& Nelson 2008; D'Angelo \& Marzari 2012; Pierens et al. 2014). However, the scale of radial migration of Jupiter and Saturn in our scenario could be less specific and much smaller than that in the Grand Tack scenario, where Jupiter and Saturn migrated inward then outward. In our case-since it is quite unlikely that Jupiter and Saturn grew already in resonance - only an inward convergent phase of migration between the giant planets is needed to put them into a resonant configuration.

The Grand Tack and the chaotic excitation models are not contradictory models. In the Grand Tack model, some level of chaotic excitation could have operated between Jupiter's and Saturn's two-phase migration and the Nice model instability. However, since the asteroid belt is already sufficiently dynamically excited after the Grand Tack(Deienno et al. 2016) it would be unnecessary to invoke chaotic excitation. We also note that both the Grand Tack and chaotic excitation mechanisms may operate with Jupiter and Saturn in either the $3: 2$ or $2: 1$ MMR (see also Pierens et al. 2014). Of course, the 3:2 resonance has been much more carefully studied for the Grand Tack, and our results suggest that the 2:1 resonance may be favored with regards to chaotic excitation. Yet we caution that Jupiter and Saturn's configuration during the disk phase may not necessarily differentiate between the two models.

\subsection{A Chaotic Young Solar System?}

It is entirely reasonable to imagine a chaotic young solar system. Several exoplanetary systems with planets on nearresonant orbits are thought to be chaotic, such as 16 Cyg B (Holman et al. 1997), GJ876 (Rivera et al. 2010), and Kepler 36 (Deck et al. 2012). The present-day solar system is well known to be chaotic (Laskar 1989, 2003; Sussman \& Wisdom 1992). The orbits of the terrestrial planets undergo chaotic diffusion on a timescale of a few million years (Laskar 1989; Batygin et al. 2015). It is unknown whether the present-day giant planets are in a chaotic configuration; an accurate determination is precluded by uncertainties in our knowledge of the planets' orbital positions (Michtchenko \& Ferraz-Mello 2001; Hayes 2008). It is often assumed that the early solar system was characterized by regular motion of the planets (Brasser et al. 2013). Our work suggests that this may not have been the case, and that the structure of the asteroid belt is a signpost that Jupiter's and Saturn's early orbits were in fact chaotic.

We showed that a small nudge could trigger chaos in Jupiter's and Saturn's early orbits (Figure 1). Could another small nudge or accumulated effects of scattering planetesimals make the system regular again? It is important to note that when the scattering of planetesimals takes place, not only damping of eccentricity but also radial migration should be observed. This process would lead to the divergent migration of Jupiter and Saturn (and ice giants) and not necessarily sinking toward the resonant center and consequently regular orbits. Typically, a late dynamical evolution is envisioned in models of solar system evolution (Gomes et al. 2005; Levison et al. 2011) which implies Jupiter and Saturn hanging out in or very near resonances for hundreds of Myr after gas disk phase. What is required for chaotic excitation to work is simply a sufficient time interval in this phase while the giant planets remain in a chaotic state. This also depends on the chaotic configuration of Jupiter and Saturn and the setup over the disk of planetesimals (Nesvorný \& Morbidelli 2012).

Obviously, it would be computationally challenging to perform a systematic analysis to identify all dynamical configurations of Jupiter and Saturn that could excite the belt. However, we indeed found that non-resonant or temporary resonant configurations can also excite the belt. We also recognized that it is not clear why previous classical simulations of terrestrial planet formation did not find similar effects in the belt. One possibility is that the chaotic effects on asteroids have been erased or mitigated by the typical presence of large planetary embryos in the belt in classical simulations (Chambers 2001; O'Brien et al. 2006; Raymond et al. 2006; Izidoro et al. 2014, 2015b). Also, these previous simulations have preferentially considered Jupiter and Saturn near their current orbits or in 3:2 MMR (in almost circular orbits; see for example Raymond et al. 2009). In our most successful simulations of belt excitation Jupiter and Saturn have eccentricities of about 0.03-0.05; the latter is consistent with results from hydrodynamical simulations (Pierens et al. 2014).

\subsection{The Absence of "Fossilized" Kirkwood Gaps}

The Kirkwood gaps in the asteroid population are created by MMRs with Jupiter. The most prominent is the gap created by Jupiter's 3:1 MMR centered at 2.50 au in the present-day belt. The chaotic excitation of the asteroid belt likely took place before a late instability in the giant planets' orbits (the so-called Nice model; Tsiganis et al. 2005; Morbidelli et al. 2007; Levison et al. 2011). If a late instability shifted Jupiter's orbit inward by $\sim 0.2$ au (Tsiganis et al. 2005) then there should exist a fossilized gap in the asteroid belt at about 2.6 au, just exterior 

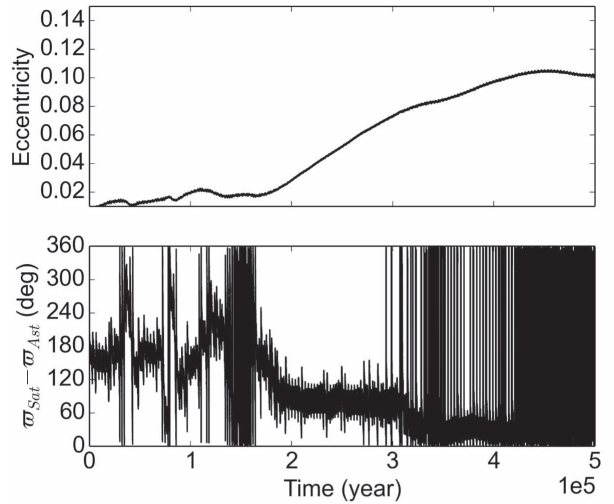

(a) Secular Resonance $\nu_{6}$
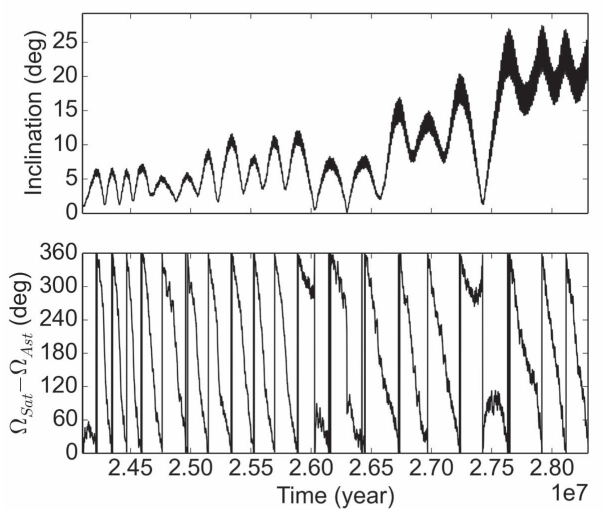

(b) Secular Resonance $\nu_{16}$

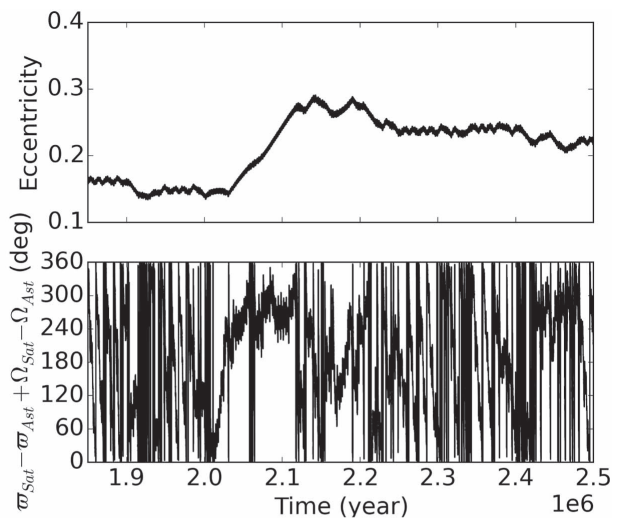

(c) Non-linear secular resonance $\nu_{6}+\nu_{16}$
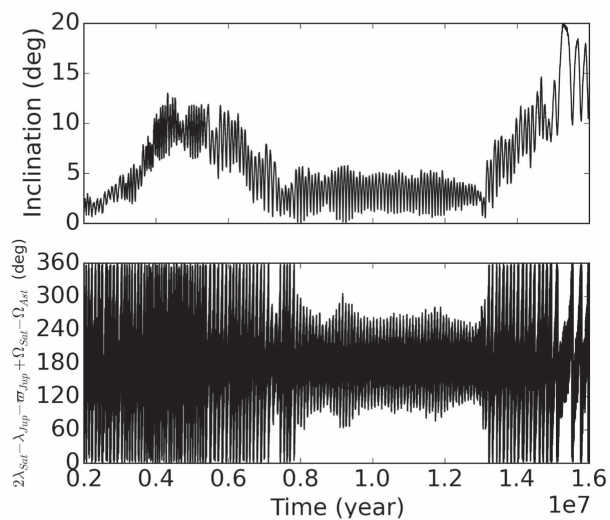

(d) Secondary Resonance
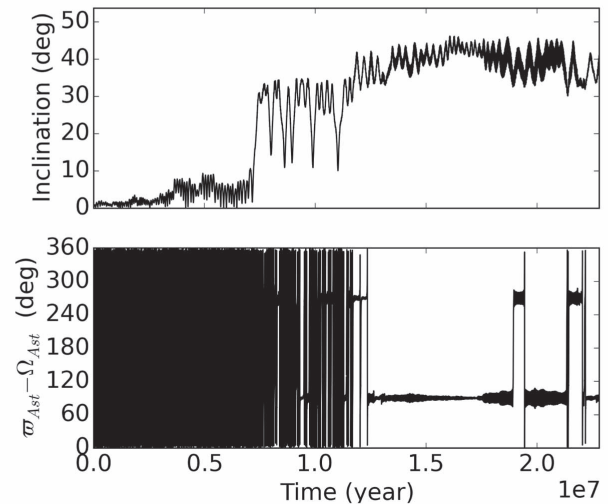

(e) Kozai Resonance

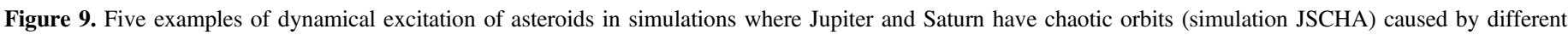
resonances. Asteroids in (a) and (b) are initially near 2 au. Asteroids in (c), (d), and (e) reside in the outer part of the main belt, between 2.65 and 3 au.

to the current Kirkwood gap associated with the 3:1 resonance. No such gap exists (see Section 6.4 of Morbidelli et al. 2010).

We caution that the data set used to study fossilized gaps (or lack thereof) are relatively sparse, containing just 335 large asteroids (with absolute magnitude $H<9.7$ ) spread across the entire main belt (see Figure 7 in Morbidelli et al. 2010). The vicinity of the 3:1 Kirkwood gap contains only $\sim 10$ asteroids. A careful statistical analysis using a larger data set (potentially extending to smaller bodies) would help quantify the depth and width of the missing Kirkwood gaps.

The belt excitation by the chaotic motion of Jupiter and Saturn seems to require an eccentricity of $\sim 0.03$ or more (although we did not perform a systematic analysis on this issue since it is beyond the scope of this paper). It was proposed by Morbidelli et al. (2010) and Deienno et al. (2016) that the pre-instability giant planets must have had almost perfectly circular orbits. Thus, Jupiter's MMRs were relatively weak and narrow such that the Kirkwood gaps before the instability were virtually nonexistent. Jupiter's eccentricity must remain extremely low $\left(e_{J}<0.01\right)$ to avoid clearing the primordial gap. This requires that during the gas disk phase the orbits of the gas giants were very efficiently damped by the gas (Morbidelli et al. 2007; Pierens et al. 2014).

The problem of the fossilized Kirkwood gaps (if it exist al all) appears to have a simple solution. During the Nice model instability, one or two ice giants are often ejected from the solar 

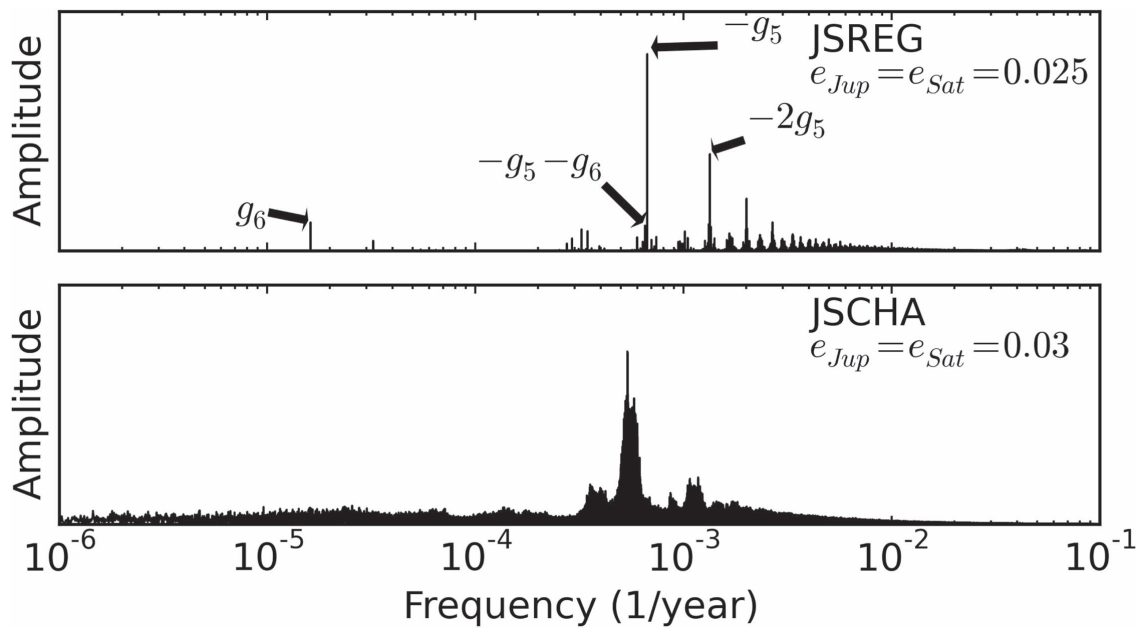

Figure 10. Frequency analysis of Jupiter's longitude of pericenter in two simulations in which Jupiter and Saturn are locked in 2:1 MMR. Top: both gas giants have initial eccentricities of 0.025 and their orbits exhibit regular motion (simulation JSREG). Bottom: the gas giants' initial eccentricities were set to 0.03 and their orbits are chaotic (simulation JSCHA). Each system was integrated numerically for $136 \mathrm{Myr}$, and to perform the Fourier analysis we have adopted an output time step of 2 years.
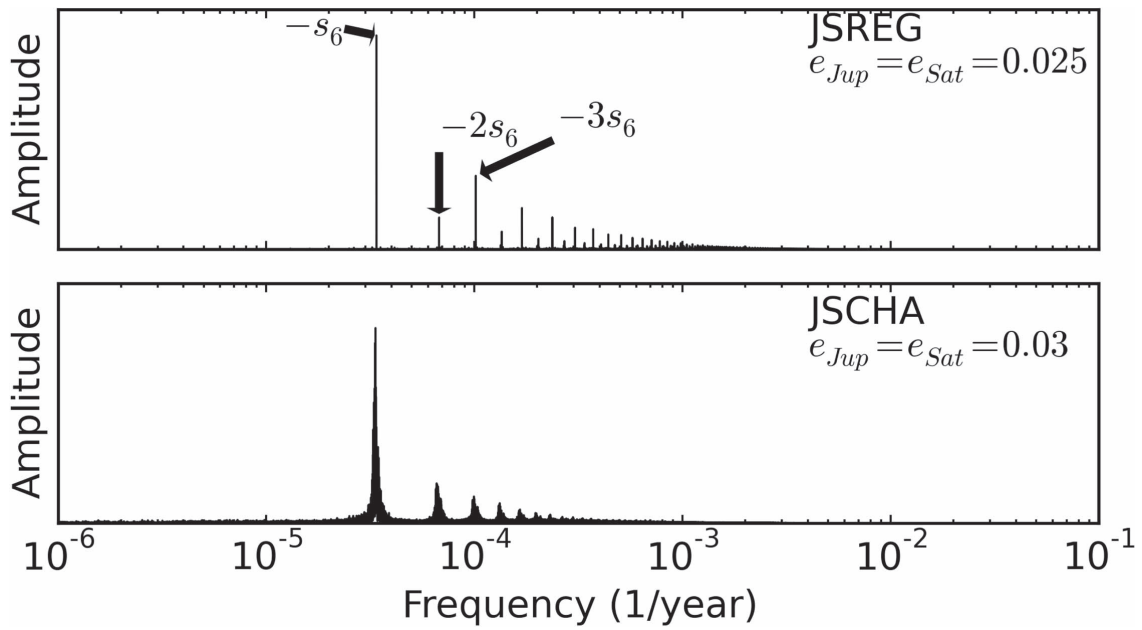

Figure 11. Frequency analysis of Jupiter's longitude of the ascending node in two simulations in which Jupiter and Saturn are locked in 2:1 MMR. Top: both gas giants have initial eccentricities of 0.025 and their orbits exhibit regular motion (simulation JSREG). Bottom: the gas giants' initial eccentricities were set to 0.03 and their orbits are chaotic (simulation JSCHA). Each system was integrated numerically for $136 \mathrm{Myr}$, and to perform the Fourier analysis we have adopted an output time step of 2 years.
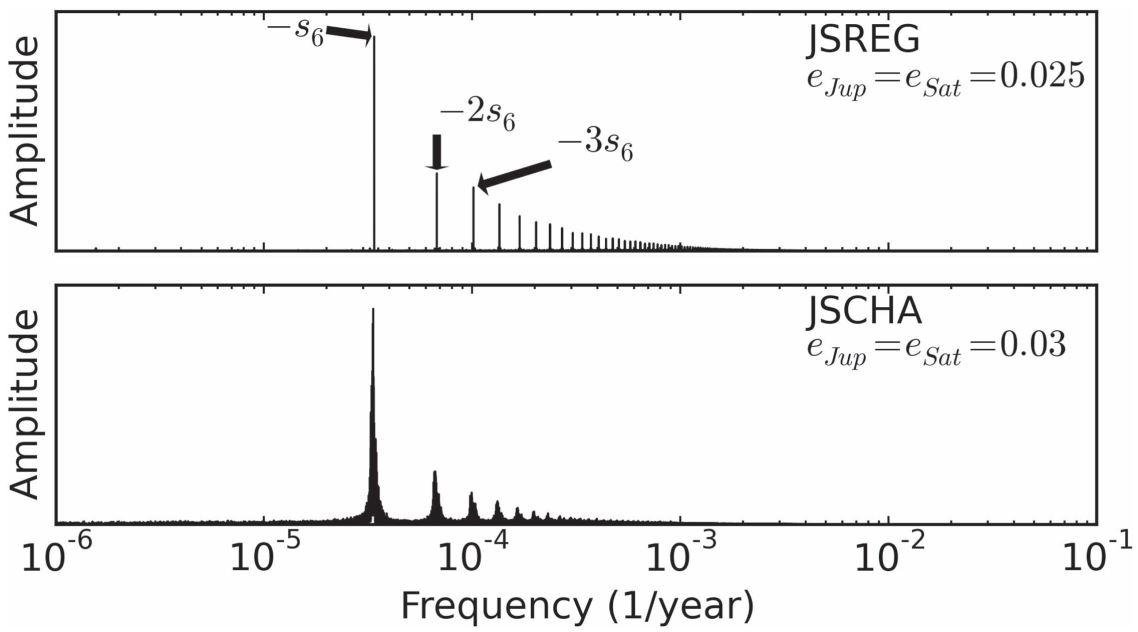

Figure 12. Frequency analysis of Saturn's longitude of the ascending node in two simulations in which Jupiter and Saturn are locked in 2:1 MMR. Top: both gas giants have initial eccentricities of 0.025 and their orbits exhibit regular motion (simulation JSREG). Bottom: the gas giants' initial eccentricities were set to 0.03 and their orbits are chaotic (simulation JSCHA). Each system was integrated numerically for $136 \mathrm{Myr}$, and to perform the Fourier analysis we have adopted an output time step of 2 years. 

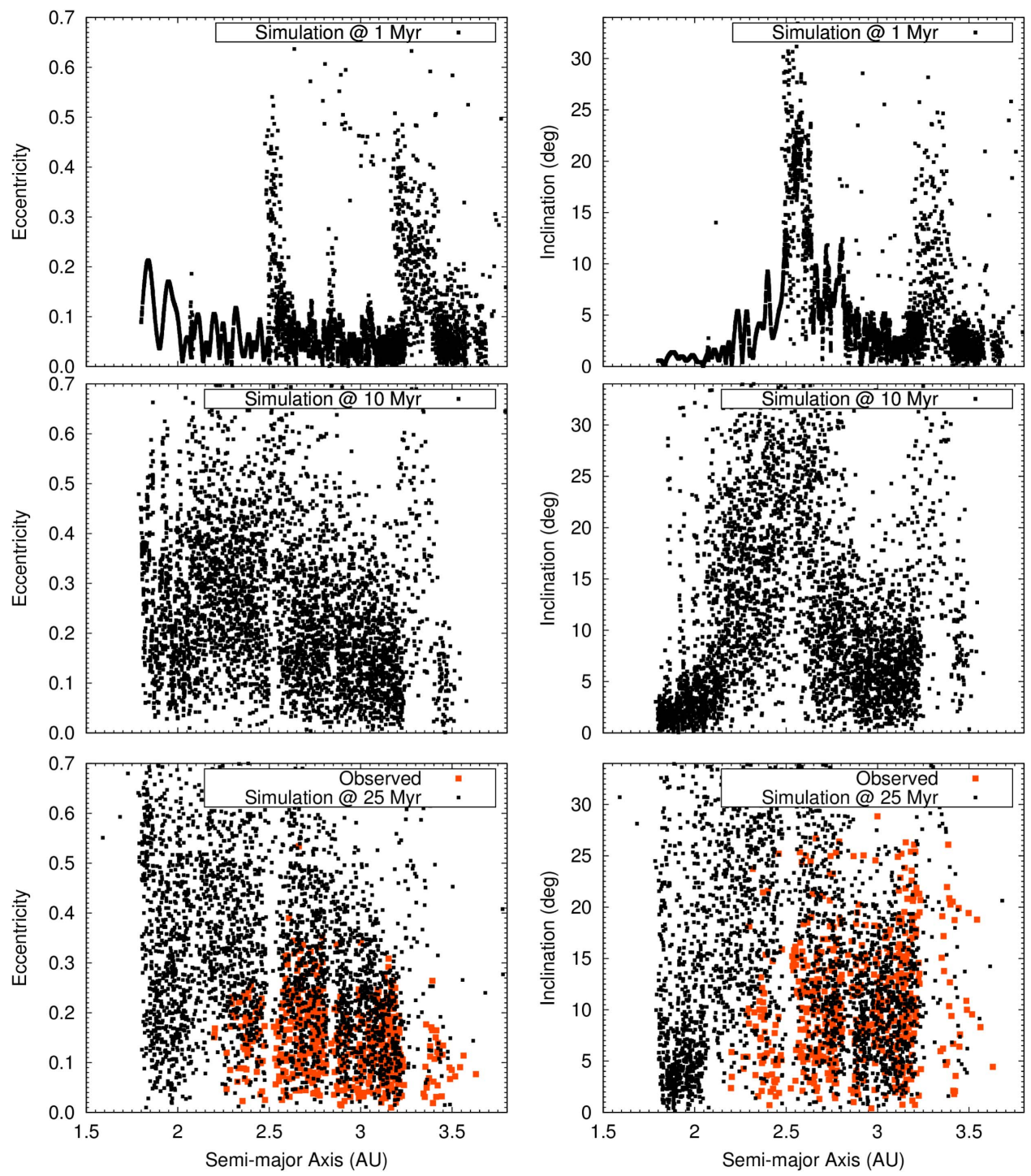

Figure 13. Dynamical evolution of asteroids in a simulation with chaotic Jupiter and Saturn in 2:1 resonance and in comparison with real asteroids with diameter larger than $50 \mathrm{~km}$.

system after suffering close encounters with Jupiter. To reproduce the solar system's current architecture, one to two additional primordial ice giants may thus have existed before the instability epoch (Nesvorny 2011; Batygin et al. 2012). During the scattering process a doomed ice giant typically spends time with an orbit interior to (but crossing) Jupiter's. The scattered ice giant passes through the asteroid belt, often crossing the main belt. While this interval is short in duration, lasting just a few tens to hundreds of thousands of years, a scattered ice giant perturbs the distribution of asteroids. Brasil et al. (2016) showed that local groupings of asteroids analogous to asteroid families, initially strongly confined in orbital parameter space, are smeared out by the scattered ice giant (see their Figures 4 and 5). This smearing would fill in any fossilized Kirkwood gaps. In the context of this evolution of the giant planets, the present-day Kirkwood gaps must have been created after the Nice model instability.

In summary, we expect that any fossilized Kirkwood gaps would have been erased by the Nice model instability (Brasil et al. 2016). The absence of fossilized gaps cannot be used as a constraint on the giant planets' early orbits.

\subsection{Implantation of C-type Asteroids in the Belt}

In this paper we did not address another important characteristic of the asteroid belt: the radial mixing of different taxonomic types of asteroids. The inner part of the main belt is dominated by S-type (water-poor) bodies, while C-type (waterrich) ones are preferentially found in the outer part of the belt, mostly beyond 2.5 au (Gradie \& Tedesco 1982; DeMeo \& Carry 2014). We demonstrate in an upcoming paper that the belt's chemical dichotomy is a natural, unavoidable outcome of the gas giants' growth in the gaseous protoplanetary disk. During the gas-accretion phase, Jupiter's core (and Saturn's as well) destabilizes the orbits of nearby small bodies and implant a fraction of these bodies in the outer asteroid belt (Izidoro et al. 2016, S. N. Raymond \& A. Izidoro 2016, in preparation). These results together with those already presented here will 

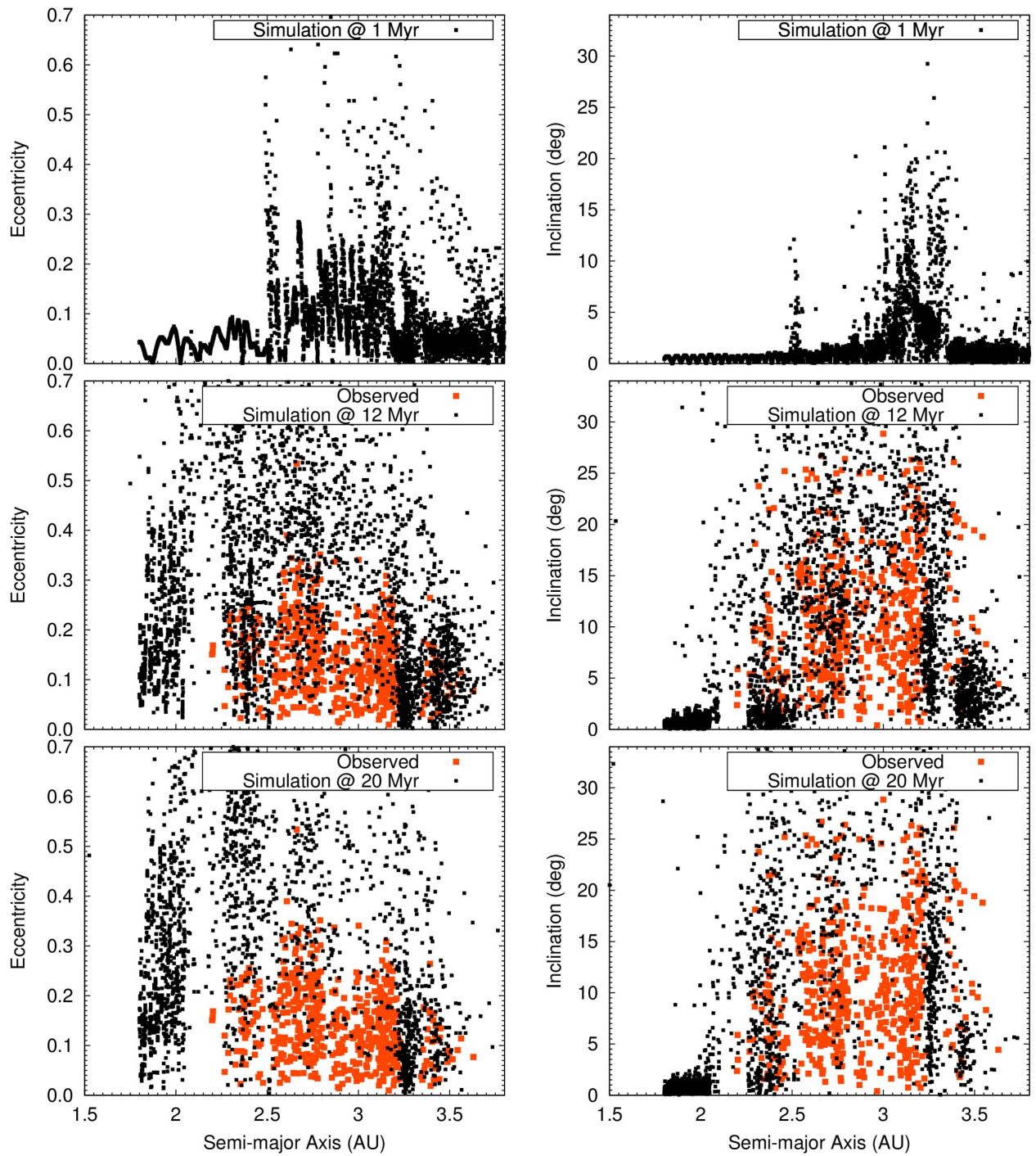

Figure 14. Dynamical evolution of asteroids in simulations with chaotic Jupiter and Saturn and in comparison with real asteroids with diameter larger than $50 \mathrm{~km}$. The period ratio between Jupiter and Saturn is about 1.75 (one of the resonant angles associated with the 7:4 resonance librate and circulate, showing that the planets are near the resonance separatrix).

form the basis for a new model to explain the bulk structure of the asteroid belt.

\section{CONCLUSIONS}

We have proposed a new mechanism to explain the puzzling orbital excitation of the asteroid belt. This mechanism requires that Jupiter's and Saturn's primordial orbits were chaotic, and we showed that this is indeed a plausible outcome of their growth and migration. The eccentricities and inclinations of asteroids are excited as a multitude of resonances stochastically jump across the full width of the belt. This mechanism is consistent with observations and has important implications for our understanding of the early solar system.

While this paper explained the orbital distribution of the asteroids, we did not explain another important feature, the taxonomic mixing of the asteroid belt. We reassure the concerned reader that we have a separate novel mechanism to explain this, which will be addressed in an upcoming paper (Izidoro et al. 2016; S. N. Raymond \& A. Izidoro 2016, in preparation).
We are very grateful to the referee, Jean-Marc Petit, for his valuable comments that helped to improve an earlier version of this paper. A.I., S.N.R., A.P., and A.M. thank the Agence Nationale pour la Recherche for support via grant ANR-13BS05-0003 (project MOJO). O.C.W. thanks FAPESP (proc. 2011/08171-3) and CNPq (proc 312813/2013-9) for financial support. Computer time for this study was provided by the computing facilities MCIA (Mésocentre de Calcul Intensif Aquitain) of the Université de Bordeaux and of the Université de Pau et des Pays de l'Adour. We are also very grateful to the CRIMSON team for managing the mesocentre SIGAMM of OCA where a significant part of these simulations were performed.

\section{APPENDIX \\ ADDITIONAL SIMULATIONS}

In this Appendix we provide extra details and address one observational constraint. First we present examples of additional resonances that act to chaotically excite the belt in the JSCHA simulation. We then present other examples of the belt's excitation for different chaotic giant planet configurations. 

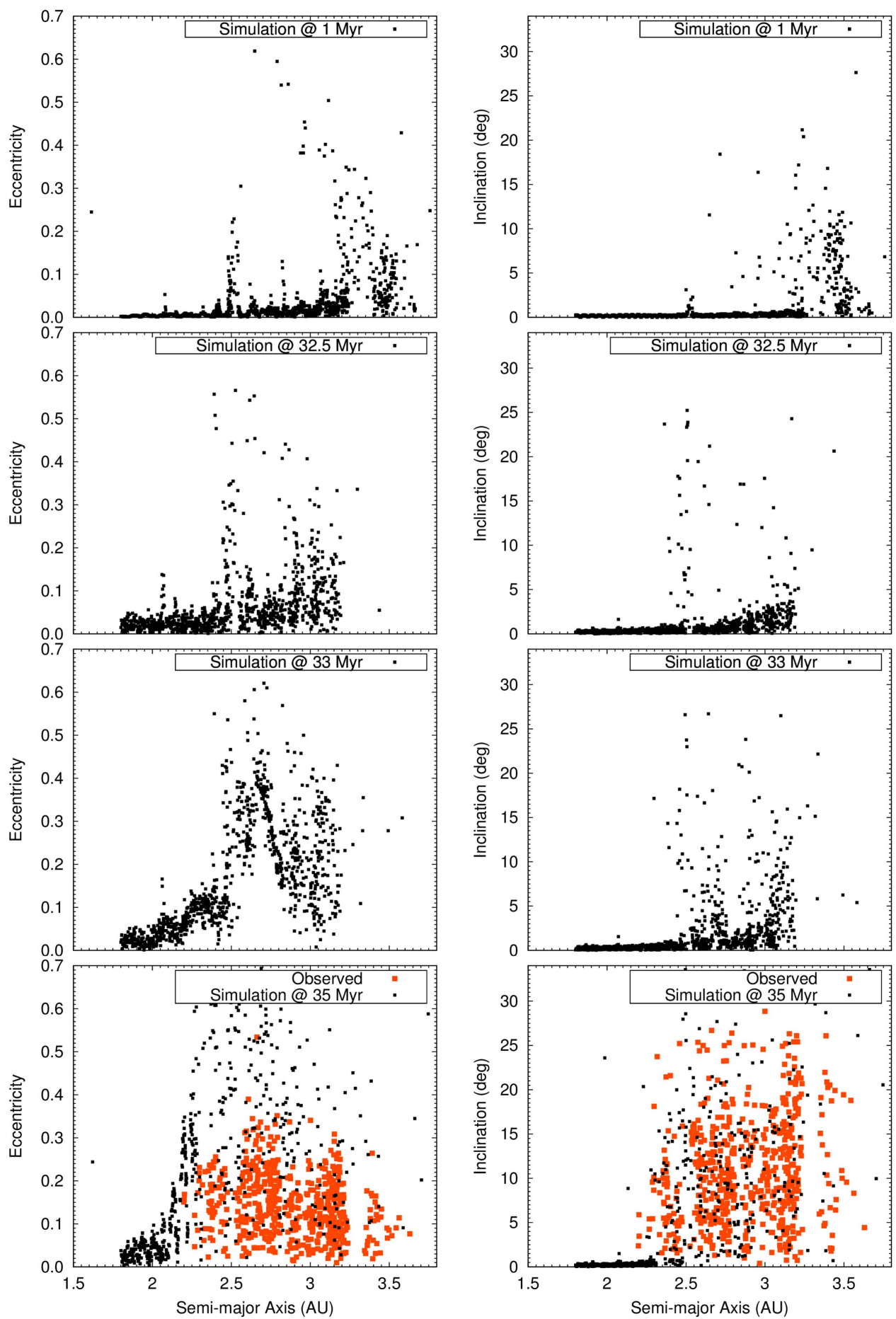

Figure 15. Dynamical evolution of asteroids in a simulation with chaotic Jupiter and Saturn and comparison with real asteroids with diameter larger than $50 \mathrm{~km}$. The period ratio between Jupiter and Saturn is about 1.66 (one of the resonant angles associated with the 5:3 resonance librate and circulate, showing that the planets are near the 5:3 resonance separatrix).

\section{A.1. The Role of Different Resonances}

Here we provide more details about the nature of the chaotic excitation mechanism by showing how different chaotically jumping resonances can excite asteroids' eccentricities and/or inclinations.

Figure 9 show examples of asteroids being excited by the perturbation of chaotic Jupiter and Saturn. These asteroids are in the same simulation as those shown in Figure 3, but are simply in different parts of the main belt. Different resonances act to increase the eccentricities and inclinations of bodies in the belt.

Figures 10-12 show the Fourier analysis of other angles and comparison between the JSREG and JSCHA simulations. The $\nu_{16} \mathrm{SR}$ is much stronger and wider in the JSCHA simulation than the JSREG simulation ( $\nu_{16}$ appears at $\sim 2.5 \mathrm{au}$; see Figures 4, 5, and 11). However, its effects in the JSCHA simulation are much more localized than those of $\nu_{6}$ in the sense of its power to affect bodies over the whole belt (if bodies have the same proper inclination and eccentricity). We did not 

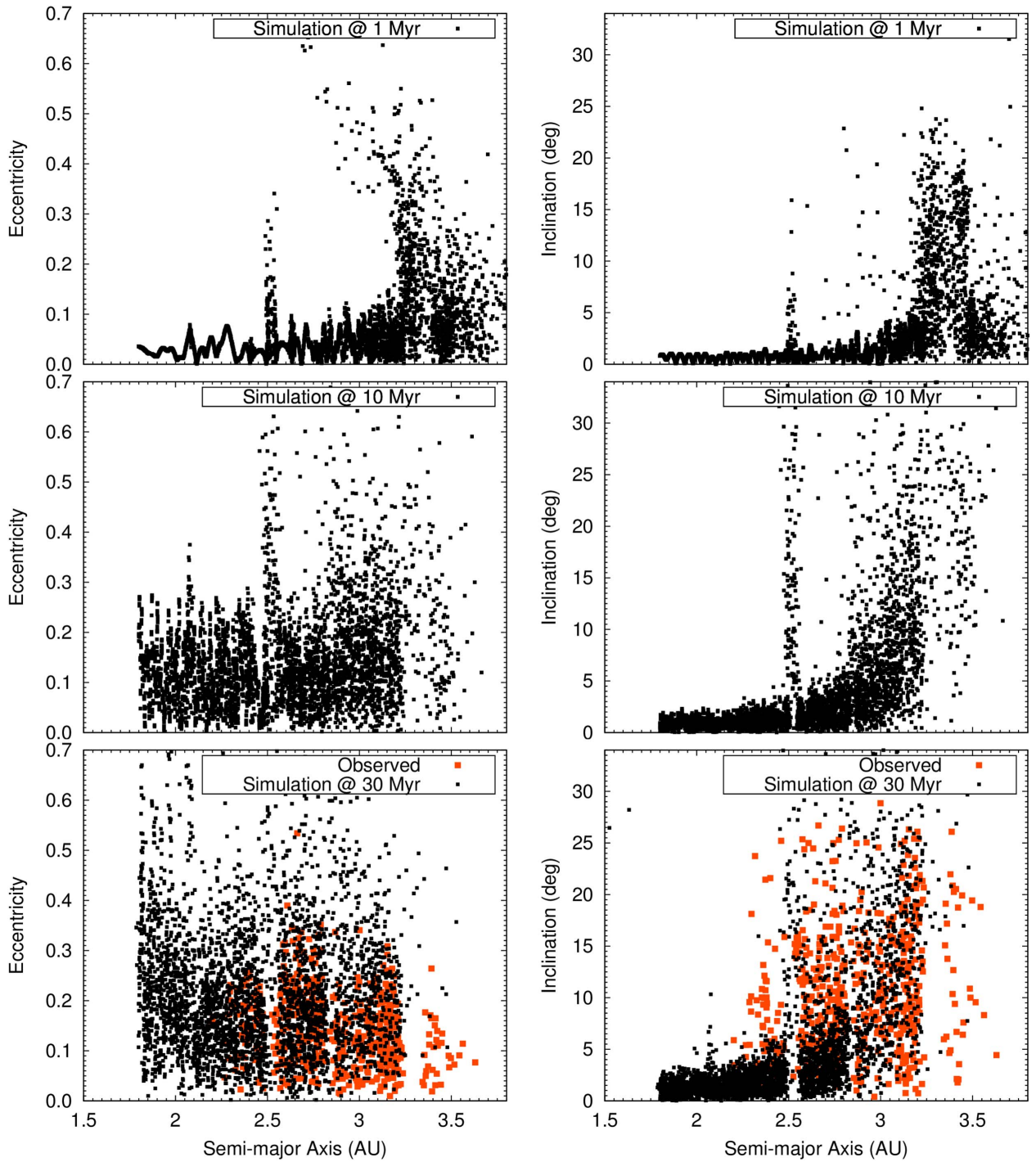

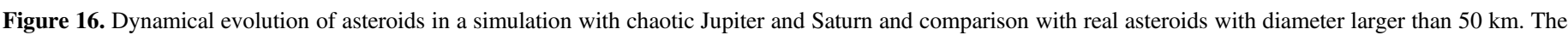

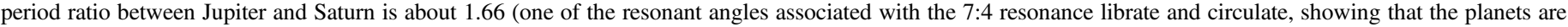
near the resonance separatrix but episodes of libration in the $3: 2$ resonance are also observed).

perform a systematic analysis of all resonances that contribute to pump inclination of bodies in the whole belt but high order secular and secondary resonances also play an important role for exciting bodies residing away from $\nu_{16}$ in the JSCHA simulation. Perhaps even three body resonances contribute. We stress that the nature of the resonances that contribute to excite bodies in the belt and their strength depends on the chaotic evolution of Jupiter and Saturn.

\section{A.2. Additional Simulations of Chaotic Excitation of the Asteroid Belt}

In this section we present several examples of dynamical excitation of the belt by different orbital configurations between Jupiter and Saturn. In these simulations we used 8000 test particles (in some cases 2000) uniformly distributed between 1.8 and $4.5 \mathrm{au}$. We stress that not all our simulations where Jupiter and Saturn had chaotic orbits successfully excited the belt. Rather, we found a spectrum of outcomes. Some simulations were not able to excite parts of the belt while in other cases the perturbations from Jupiter and Saturn were so strong that the belt was destroyed. We did not perform a systematic analysis looking for the optimal resonant or nonresonant chaotic configuration to excite the belt. However, we have used as fiducial case a dynamical configuration where Jupiter and Saturn are in 2:1 MMR.

Figure 13 shows the dynamical evolution of asteroids in a simulation with chaotic Jupiter and Saturn in 2:1 resonance. Figures 14-17 show the dynamical evolution of asteroids in the belt in simulations where Jupiter and Saturn are in different resonant configurations, with period ratios of $\sim 1.75, \sim 1.66$, $\sim 1.66$, and $\sim 1.5$, respectively. Simulations were integrated using Symba (Duncan et al. 1998) with a 0.1 year time step. The total duration of each simulation varied between 20 and $125 \mathrm{Myr}$, and each simulation's duration is indicated in the bottom panel. Figures 13, 14, and 16 show simulations initially with 8000 test particles. Simulations corresponding to Figures 15 and 17 initially contain 2000 test particles.

Figure 14 shows a simulation in which the whole belt was excited both in eccentricity and inclination. However, some regions of the belt were overly depleted compared with others after $20 \mathrm{Myr}$ of integration. 

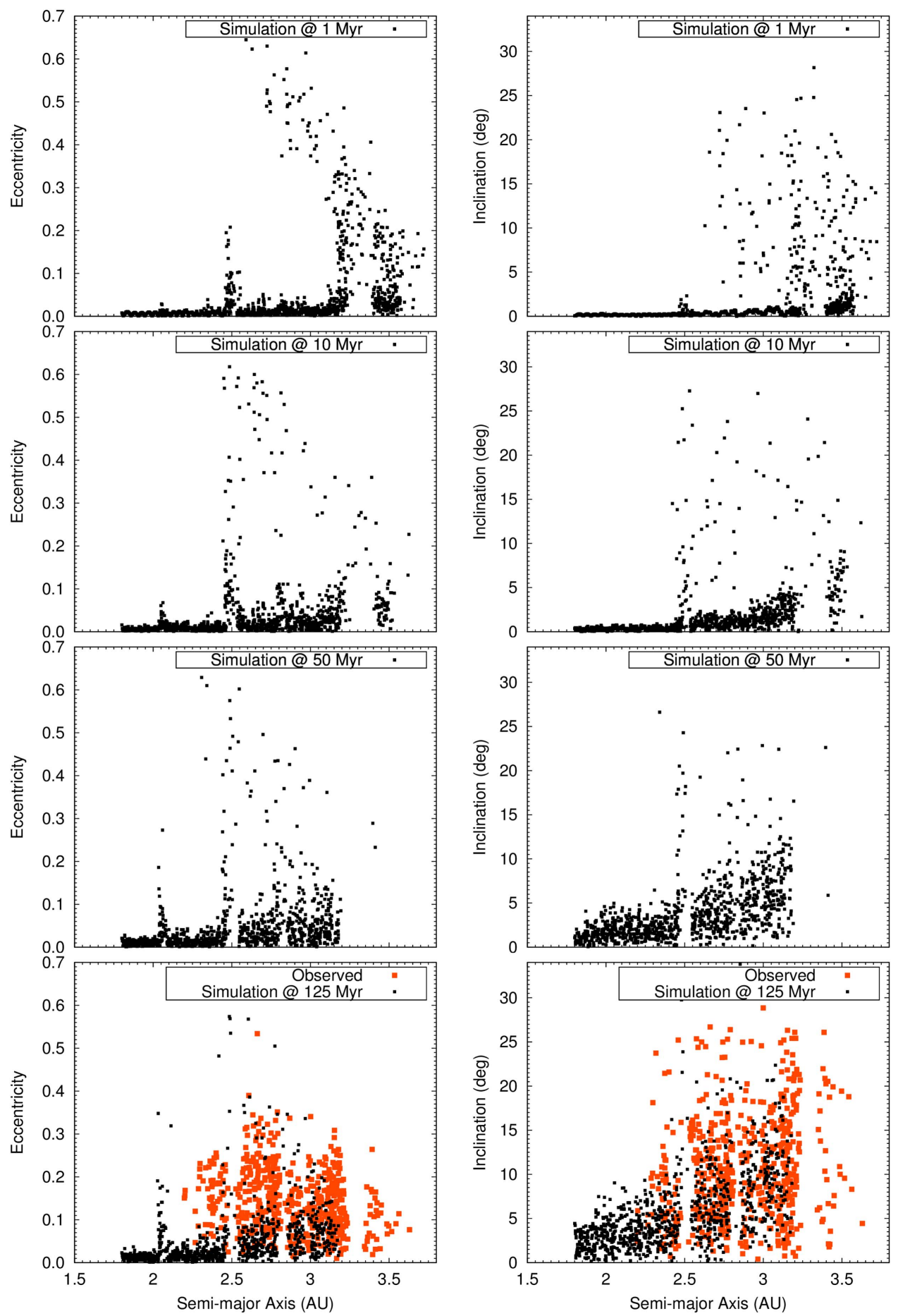

Figure 17. Dynamical evolution of asteroids in a simulation with chaotic Jupiter and Saturn and comparison with real asteroids with diameter larger than $50 \mathrm{~km}$. The period ratio between Jupiter and Saturn is about 1.5 (one of the resonant angles associated with the 3:2 resonance librate and circulate, showing that the planets are near the resonance separatrix).

Figure 15 is a very interesting case. Asteroids in the belt maintained low orbital inclinations and eccentricities for more than 30 Myr. At $\sim 33$ Myr a stochastic jump in the positions of the giant planets (a consequence of their orbits being chaotic) resulted in a very strong perturbation in the belt (see corresponding panel in Figure 15). Within $2 \mathrm{Myr}$ of the jump, the whole belt was excited to the observed levels of the real one. This case shows that excitation of the entire belt may be an extremely fast event. However, typically, the complete belt excitation seems to require about $\sim 10 \mathrm{Myr}$ or so.
Figure 16 shows a case where the level of eccentricity excitation is consistent with the observed one. However, the region between 2.1 and 2.8 au is under-excited in inclination. In general an under-excited inner belt is less of a problem than an under-excited outer belt, because perturbations from the inner parts of the solar system (e.g., Mars and other remnant planetary embryos) may excite the inner belt but not the outer belt (Izidoro et al. 2015b).

Figure 17 shows a simulation where Jupiter and Saturn are near the 3:2 resonance. In this case, both eccentricities and 
Table 1

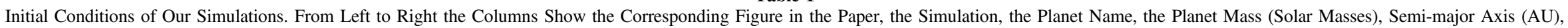
Eccentricity, Orbital Inclination (DEG.), Argument of Pericenter (DEG.), Longitude of the Ascending Node (DEG.) and Mean Anomaly (DEG.)

\begin{tabular}{|c|c|c|c|c|c|c|c|c|c|}
\hline Figure & Simulation & Planet & Mass $\left(\mathrm{M}_{\odot}\right)$ & Semi-major Axis (AU) & Eccentricity & Inclination (deg.) & $\begin{array}{c}\text { Argument of } \\
\text { Pericenter (deg.) }\end{array}$ & $\begin{array}{l}\text { Longitude of the ascending } \\
\text { node (deg.) }\end{array}$ & Mean Anomaly (deg.) \\
\hline \multicolumn{10}{|c|}{ Figure 1} \\
\hline & & Jupiter & $10^{-3}$ & 5.25 & 0.025 & 0 & 0 & 0 & 0 \\
\hline & & Saturn & $2.85 \times 10^{-4}$ & 8.33385552283305 & 0.025 & 0.5 & 0 & 0 & 180 \\
\hline & & Embryo & $3.0 \times 10^{-7}$ & 12 & 0.035 & 0 & 0 & 0 & 250 \\
\hline \multicolumn{10}{|c|}{ Figure 2} \\
\hline & JSREG & Jupiter & $10^{-3}$ & 5.4 & 0.025 & 0 & 0 & 0 & 0 \\
\hline & JSREG & Saturn & $2.85 \times 10^{-4}$ & 8.5719656 & 0.025 & 0.5 & 0 & 0 & 180 \\
\hline & JSCHA & Jupiter & $10^{-3}$ & 5.4 & 0.03 & 0 & 0 & 0 & 0 \\
\hline & JSCHA & Saturn & $2.85 \times 10^{-4}$ & 8.5719656 & 0.03 & 0.5 & 0 & 0 & 180 \\
\hline \multicolumn{10}{|c|}{ Figure 4} \\
\hline & JSREG & Jupiter & $10^{-3}$ & 5.25 & 0.025 & 0 & 0 & 0 & 0 \\
\hline & JSREG & Saturn & $2.85 \times 10^{-4}$ & 8.33385552283305 & 0.025 & 0.5 & 0 & 0 & 180 \\
\hline & JSCHA & Jupiter & $10^{-3}$ & 5.25 & 0.03 & 0 & 0 & 0 & 0 \\
\hline & JSCHA & Saturn & $2.85 \times 10^{-4}$ & 8.33385552283305 & 0.03 & 0.5 & 0 & 0 & 180 \\
\hline \multicolumn{10}{|c|}{ Figure 13} \\
\hline & & Jupiter & $10^{-3}$ & 5.25 & 0.00324129 & 0 & 0 & 0 & 0 \\
\hline & & Saturn & $2.85 \times 10^{-4}$ & 8.34031296 & 0.03543211 & 1.33660054 & 338.16098022 & 203.47100830 & 178.91156006 \\
\hline \multicolumn{10}{|c|}{ Figure 14} \\
\hline & & Jupiter & $10^{-3}$ & 5.25 & 0.00115474 & 0 & 0 & 0 & 0 \\
\hline & & Saturn & $2.85 \times 10^{-4}$ & 7.64275074 & 0.04558534 & 1.20436156 & 215.75094604 & 282.47793579 & 155.35372925 \\
\hline \multicolumn{10}{|c|}{ Figure 15} \\
\hline & & Jupiter & $10^{-3}$ & 5.25 & 0.00397692 & 0 & 0 & 0 & 0 \\
\hline & & Saturn & $2.85 \times 10^{-4}$ & 7.33959627 & 0.01606708 & 0.48118880 & 258.75650024 & 358.17221069 & 160.78182983 \\
\hline \multicolumn{10}{|c|}{ Figure 16} \\
\hline & & Jupiter & $10^{-3}$ & 5.25 & 0.00127964 & 0 & 0 & 0 & 0 \\
\hline & & Saturn & $2.85 \times 10^{-4}$ & 7.39253807 & 0.02013579 & 1.65362096 & 250.55947876 & 167.58595276 & 31.68255806 \\
\hline \multicolumn{10}{|c|}{ Figure 17} \\
\hline & & Jupiter & $10^{-3}$ & 5.25 & 0.00292543 & 0 & 0 & 0 & 0 \\
\hline & & Saturn & $2.85 \times 10^{-4}$ & 6.83152723 & 0.02512858 & 0.42025852 & 288.54019165 & 166.67124939 & 291.39468384 \\
\hline
\end{tabular}


orbital inclination of bodies in the belt are modestly underexcited when compared with the real belt.

Despite some cases failing to reproduce the dynamical excitation of the belt, we stress that in all these examples the level of dynamical excitation produced is substantially higher than if Jupiter and Saturn had those respective period ratios but regular orbits. When Jupiter and Saturn have regular orbits, only objects near strong SRs (as $\nu_{6}$ and $\nu_{16}$ ) and MMRs have their eccentricities and inclinations significantly increased relative to the initial value (almost coplanar and circular orbits; (Raymond et al. 2009; Izidoro et al. 2015b)).

\section{A.3. Initial Conditions of our Main Simulations}

In this section (see Table 1) we provide the initial conditions of our simulations corresponding to Figures 1, 2, 4, and 13-17.

\section{REFERENCES}

Adams, F., Laughlin, G., \& Bloch, A. 2008, ApJ, 683, 1117

Agnor, C. B., Canup, R. M., \& Levison, H. F. 1999, Icar, 142, 219

Barnes, R., Deitrick, R., Greenberg, R., Quinn, T. R., \& Raymond, S. N. 2015 , ApJ, 801, 101

Baruteau, C., Crida, A., Paardekooper, S.-J., et al. 2014, in Protostars Planets VI, ed. H. Beuther et al. (Tucson, AZ: Univ. Arizona Press), 667

Batygin, K., Brown, M. E., \& Betts, H. 2012, ApJL, 744, L3

Batygin, K., \& Morbidelli, A. 2013, A\&A, 556, A28

Batygin, K., Morbidelli, A., \& Holman, M. J. 2015, ApJ, 799, 120

Bitsch, B., Crida, A., Libert, A.-S., \& Lega, E. 2013, A\&A, 555, A124

Bottke, W. F., Walker, R. J., Day, J. M. D., Nesvorny, D., \& Elkins-Tanton, L. 2010, Sci, 330, 1527

Brasil, P., Roig, F., Nesvorný, D., et al. 2016, Icar, 266, 142

Brasser, R., Matsumura, S., Ida, S., Mojzsis, S. J., \& Werner, S. C. 2016, ApJ, 821,75

Brasser, R., Walsh, K. J., \& Nesvorńy, D. 2013, MNRAS, 433, 3417

Chambers, J. E. 1999, MNRAS, 304, 793

Chambers, J. E. 2001, Icar, 152, 205

Chambers, J. E., \& Wetherill, G. W. 1998, Icar, 136, 304

Cincotta, P. M., \& Simo, C. 2000, A\&AS, 147, 205

Cresswell, P., \& Nelson, R. P. 2008, A\&A, 482, 677

Cresswell, P., \& Nelson, R. P. 2006, A\&A, 450, 833

Crida, A., Sandor, Z., \& Kley, W. 2008, A\&A, 483, 325

D’Angelo, G., \& Marzari, F. 2012, ApJ, 757, 50

Day, J. M. D., Pearson, D. G., \& Taylor, L. a. 2007, Sci, 315, 217

Deck, K. M., Holman, M. J., Agol, E., et al. 2012, ApJL, 755, L21

Deienno, R., Gomes, R. S., Walsh, K. J., Morbidelli, A., \& Nesvorný, D. 2016, Icar, 272, 114

DeMeo, F. E., \& Carry, B. 2014, Natur, 505, 629

de Val-Borro, M., Edgar, R. G., Artymowicz, P., et al. 2006, MNRAS, 370, 529

Drazkowska, J., Alibert, Y., \& Moore, B. 2016, arXiv:1607.05734

Duncan, M. J., Levison, H. F., \& Lee, M. H. 1998, AJ, 116, 2067

Fischer, R. A., \& Ciesla, F. J. 2014, E\&PSL, 392, 28

Froeschle, C., \& Scholl, H. 1989, CeMDA, 46, 231

Gomes, R., Levison, H. F., Tsiganis, K., \& Morbidelli, A. 2005, Natur, 435, 466

Gradie, J., \& Tedesco, E. 1982, Sci, 216, 1405

Hayashi, C. 1981, PThPS, 70, 35

Hayes, W. B. 2008, MNRAS, 386, 295

Holman, M., Touma, J., \& Tremaine, S. 1997, Natur, 386, 254

Izidoro, A., de Souza Torres, K., Winter, O. C., \& Haghighipour, N. 2013, ApJ, 767, 54

Izidoro, A., Haghighipour, N., Winter, O. C., \& Tsuchida, M. 2014, ApJ, 782,31

Izidoro, A., Morbidelli, A., Raymond, S. N., Hersant, F., \& Pierens, A. 2015a, A\&A, 582, A99

Izidoro, A., Raymond, S. N., Morbidelli, A., \& Winter, O. C. 2015b, MNRAS, 453, 3619

Izidoro, A., Raymond, S. N., Pierens, A., et al. 2016, American Astronomical Society DDA Meeting \#47, 300.03

Jacobson, S. A., \& Morbidelli, A. 2014, RSPTA, 372, 20130174

Jacobson, S. A., Morbidelli, A., Raymond, S. N., et al. 2014, Natur, 508, 84

Johansen, A., Blum, J., Tanaka, H., et al. 2014, in Protostars and Planets VI, ed. H. Beuther et al. (Tucson, AZ: Univ. Arizona Press), 547

Kaib, N. A., \& Chambers, J. E. 2016, MNRAS, 455, 3561
Laskar, J. 1989, Natur, 338, 237

Laskar, J. 1990, Icar, 88, 266

Laskar, J. 1993, CeMDA, 56, 191

Laskar, J. 2003, AnHP, 4, 693

Lecoanet, D., Adams, F. C., \& Bloch, A. M. 2009, ApJ, 692, 659

Levison, H., \& Duncan, M. J. 1994, Icar, 108, 18

Levison, H. F., Kretke, K. A., Walsh, K. J., \& Bottke, W. F. 2015, PNAS, 112 , 14180

Levison, H. F., Morbidelli, A., Tsiganis, K., Nesvorný, D., \& Gomes, R. 2011, AJ, 142, 152

Lin, D. N. C., \& Papaloizou, J. 1986, ApJ, 309, 846

Lykawka, P. S., \& Ito, T. 2013, ApJ, 65, 65

Masset, F., \& Snellgrove, M. 2001, MNRAS, 320, L55

Michtchenko, T. A., Beaugé, C., \& Ferraz-Mello, S. 2008, MNRAS, 387, 747

Michtchenko, T. A., \& Ferraz-Mello, S. 1995, A\&A, 303, 945

Michtchenko, T. A., \& Ferraz-Mello, S. 2001, AJ, 122, 474

Minton, D. A., \& Malhotra, R. 2010, Icar, 207, 744

Morbidelli, A., Brasser, R., Gomes, R., Levison, H. F., \& Tsiganis, K. 2010, AJ, 140, 1391

Morbidelli, A., \& Crida, A. 2007, Icar, 191, 158

Morbidelli, A., \& Henrard, J. 1991, CeMDA, 51, 169

Morbidelli, A., Tsiganis, K., Crida, A., Levison, H. F., \& Gomes, R. 2007, AJ, 134, 1790

Morbidelli, A., Walsh, K. J., O’Brien, D. P., Minton, D. A., \& Bottke, W. F. 2015, in Asteroids IV, ed. P. Michel, F. E. DeMeo, \& W. F. Bottke (Tucson, AZ: Univ. Arizona Press), 493

Moriarty, J., \& Fischer, D. 2015, ApJ, 809, 94

Morishima, R., Schmidt, M. W., Stadel, J., \& Moore, B. 2008, ApJ, 685, 1247

Murray, C. D., \& Dermott, S. F. 1999, Solar System Dynamics (Cambridge: Cambridge Univ. Press)

Nesvorny, D. 2011, ApJL, 742, L22

Nesvorný, D., \& Morbidelli, A. 2012, AJ, 144, 117

O’Brien, D. P., Morbidelli, A., \& Bottke, W. F. 2007, Icar, 191, 434

O'Brien, D. P., Morbidelli, A., \& Levison, H. F. 2006, Icar, 184, 39

Papaloizou, J. C. B., \& Larwood, J. D. 2000, MNRAS, 315, 823

Petit, J.-M., Chambers, J., Franklin, F., \& Nagasawa, M. 2002, in Asteroids III ed. W. F. Bottke, Jr. et al. (Tucson, AZ: Univ. Arizona Press), 711

Petit, J.-M., Morbidelli, A., \& Chambers, J. 2001, Icar, 153, 338

Petit, J.-M., Morbidelli, A., \& Valsecchi, G. B. 1999, Icar, 141, 367

Pierens, A., Baruteau, C., \& Hersant, F. 2011, A\&A, 531, A5

Pierens, A., \& Nelson, R. P. 2008, A\&A, 482, 333

Pierens, A., \& Raymond, S. N. 2011, A\&A, 533, 131

Pierens, A., Raymond, S. N., Nesvorny, D., \& Morbidelli, A. 2014, ApJL, 795, L11

Raymond, S. N., \& Morbidelli, A. 2014, in IAU Symp. 310, Complex Planetary Systems, ed. Z. Knežević \& A. Lemaitre (Cambridge: Cambridge Univ. Press), 194

Raymond, S. N., O’Brien, D. P., Morbidelli, A., \& Kaib, N. A. 2009, Icar, 203, 644

Raymond, S. N., Quinn, T., \& Lunine, J. I. 2004, Icar, 168, 1

Raymond, S. N., Quinn, T., \& Lunine, J. I. 2006, Icar, 183, 265

Rein, H., \& Spiegel, D. S. 2014, MNRAS, 446, 1424

Rein, H., \& Tamayo, D. 2015, MNRAS, 452, 376

Rivera, E. J., Laughlin, G., Butler, R. P., et al. 2010, ApJ, 719, 890

Roig, F., \& Nesvorný, D. 2015, AJ, 150, 186

Sándor, Z., \& Kley, W. 2006, A\&A, 451, L31

Shakura, N. I., \& Sunyaev, R. A. 1973, A\&A, 24, 337

Surville, C., Mayer, L., \& Lin, D. N. C. 2016, arXiv:1601.05945

Sussman, G. J., \& Wisdom, J. 1992, Sci, 257, 56

Tanaka, H., Takeuchi, T., \& Ward, W. R. 2002, ApJ, 565, 1257

Tanaka, H., \& Ward, W. R. 2004, ApJ, 602, 388

Tsiganis, K., Gomes, R., Morbidelli, A., \& Levison, H. F. 2005, Natur, 435, 459

Walker, R. J. 2009, ChEG, 69, 101

Walsh, K. J., Morbidelli, A., Raymond, S. N., O’Brien, D. P., \& Mandell, A. M. 2011, Natur, 475, 206

Ward, W. 1997, Icar, 126, 261

Weidenschilling, S. J. 1977, MNRAS, 180, 57

Wetherill, G. 1996, Icar, 119, 219

Wetherill, G. W. 1978, in Protostars and Planets: Studies of star formation and of the origin of the solar system, ed. T. Gehrels (Tucson, AZ: Univ. Arizona Press), 565

Wetherill, G. W. 1992, Icar, 100, 307

Wetherill, G. W. 1986, in Lunar and Planetary Institute Conf. Proc., Origin of the moon, ed. W. Hartmann, R. Phillips, \& G. Taylor (Tucson, AZ: Planetary Science Institute), 519

Zhang, H., \& Zhou, J.-L. 2010, ApJ, 714, 532 